\title{
3DICE coding matrix multidirectional macro-architecture modulates cell organization, shape, and co-cultures endothelization network
}

\author{
Raphaël F. Canadas a,b,f,*, João B. Costa ${ }^{\text {a,b }}$, Zhengwei Mao ${ }^{c}$, Changyou Gao ${ }^{c}$, Utkan Demirci ${ }^{\text {d,e }}$, \\ Rui L. Reis ${ }^{\mathrm{a}, \mathrm{b}}$, Alexandra P. Marques ${ }^{\mathrm{a}, \mathrm{b}}$, Joaquim M. Oliveira ${ }^{\mathrm{a}, \mathrm{b}},{ }^{* *}$ \\ a $3 B$ 's Research Group, I3Bs, Research Institute on Biomaterials, Biodegradables and Biomimetics, University of Minho, Headquarters of the European Institute of \\ Excellence on Tissue Engineering and Regenerative Medicine, Zona Industrial da Gandra, AvePark, Barco GMR, 4805-017, Portugal \\ ' ICVS/3B's, PT Government Associate Laboratory, Braga, Guimarães, Portugal \\ ${ }^{\mathrm{c}}$ MOE Key Laboratory of Macromolecular Synthesis and Functionalization, Department of Polymer Science and Engineering, Zhejiang University, Hangzhou, Zhejiang, \\ 310027, China \\ d Bio-Acoustic MEMS in Medicine (BAMM) Laboratory, Canary Center at Stanford for Cancer Early Detection, Department of Radiology, Stanford School of Medicine, \\ Palo Alto, CA, 94304, USA \\ ${ }^{\mathrm{e}}$ Electrical Engineering Department by Courtesy, Stanford University, Stanford, CA, 94305, USA \\ ${ }^{\mathrm{f}}$ Tech4MED ${ }^{\mathrm{TM}}$, UPTEC, ASPRELA I, Office-Lab 0.16, Business Campus, $n .{ }^{\circ} 455 / 461,4200-135$ Porto, Portugal
}

\section{A R T I C L E I N F O}

\section{Keywords:}

Cryogel

Ice-templating

Hepatic cirrhosis

Biomaterials

Tissue engineering

Regenerative medicine

\begin{abstract}
A B S T R A C T
Natural extracellular matrix governs cells providing biomechanical and biofunctional outstanding properties, despite being porous and mostly made of soft materials. Among organs, specific tissues present specialized macro-architectures. For instance, hepatic lobules present radial organization, while vascular sinusoids are branched from vertical veins, providing specific biofunctional features. Therefore, it is imperative to mimic such structures while modeling tissues. So far, there is limited capability of coupling oriented macro-structures with interconnected micro-channels in programmable long-range vertical and radial sequential orientations. Herein, a three-directional ice crystal elongation (3DICE) system is presented to code geometries in cryogels. Using 3DICE, guided ice crystals growth templates vertical and radial pores through bulky cryogels. Translucent isotropic and anisotropic architectures of radial or vertical pores are fabricated with tunable mechanical response. Furthermore, 3D combinations of vertical and radial pore orientations are coded at the centimeter scale. Cell morphological response to macro-architectures is demonstrated. The formation of endothelial segments, CYP450 activity, and osteopontin expression, as liver fibrosis biomarkers, present direct response and specific cellular organization within radial, linear, and random architectures. These results unlock the potential of ice-templating demonstrating the relevance of macro-architectures to model tissues, and broad possibilities for drug testing, tissue engineering, and regenerative medicine.
\end{abstract}

\section{Introduction}

In vivo, within the $3 \mathrm{D}$ space of the cellular microenvironment, the native extracellular matrix (ECM) is highly heterogeneous, and cellular alignment occurs [1] [-] [3]. Once most of the tissues components have multidimensional order and orientation, the multidirectional macro-architecture requires mimicry to achieve and understand tissue function [4] [-] [7]. For instance, the liver is a typical tissue of multidirectional stratified architecture, composed of sets of hepatic lobules, which presents vertical and radial cellular arrangements, comprising vasculature and multiple cell types [8] [-] [10].

Thus, multidirectional macro-architecture is an important aspect to model tissues and organs for pharmaceutical testing, clinical implantation, or fundamental research. So, these micro-organ structures should match the dimensional properties of the in vivo tissue at the in vitro setting $[1,11,12]$. So far, it is well known that cells can align along with

\footnotetext{
* Corresponding author. 3B's Research Group, I3Bs, Research Institute on Biomaterials, Biodegradables and Biomimetics, University of Minho, Headquarters of the European Institute of Excellence on Tissue Engineering and Regenerative Medicine, Zona Industrial da Gandra, AvePark, Barco GMR, 4805-017, Portugal.

** Corresponding author. 3B's Research Group, I3Bs, Research Institute on Biomaterials, Biodegradables and Biomimetics, University of Minho, Headquarters of the European Institute of Excellence on Tissue Engineering and Regenerative Medicine, Zona Industrial da Gandra, AvePark, Barco GMR, 4805-017, Portugal.

E-mail addresses: raphael.canadas@tech4med.pt (R.F. Canadas), miguel.oliveira@i3bs.uminho.pt (J.M. Oliveira).
} 
micro- and nanosized parallel grooves/ridges [13] [-] [15] when the periodicity and dimensions of the patterns are above $100 \mathrm{~nm}$ groove depth [16]. Moreover, several studies reported that micro- and nanopatterned structures can be a valuable tool for directed growth $[17,18]$ and differentiation of cells into specific tissue-lineages on 2D [19,20], or in 3D architectures [6,21]. Yet, reported synthetic ECM fabrication systems fail when recreating macro-scale multidirectional tissues constituted by multiple vertical and radial orientations of pores in 3D, and there is limited research on how macro-architecture (several hundreds of micrometers range) influences biological constructs in vitro, which is of major importance.

Last decade, 3D printing evolved as the most promising approach for manufacturing and coding functional bioarchitectures at the millimeter/ centimeter scale. However, this method offers limited control over 3D porous architecture, mainly when applied to nano- and micro-scale polymeric structures, and often results in printed fiber fusion or collapsing [22,23]. Therefore, there is an unmet need for an effective fabrication method with precise control on the configurability of porosity in 3D hydrogels.

A top-down templating method exploiting ice crystals nucleation and growth into multiple orientations could enable a versatile and scalable approach for the fabrication and coding of multidirectional cryogel architectures. The ice-templating technique has been previously used for the 3D fabrication of homogeneous aligned porous structures [24] [-] [26]. While low thermal conductivity molds ensure directional freezing [27], the use of high thermally conductive molds induces radial ice growth towards the mold [28]. However, the desired multidirectional architectures composed of continuous linear and radial porous zones have not been successfully developed so far, making the state-of-the-art about ice-templating technique quite limited. Therefore, innovative systems to template multiple pore orientations in 3D need to be developed in order to address in an efficient manner the architectural requirements for biomedical and clinical applications.

Herein, we report 3DICE, a versatile, efficient, and scalable device, and strategy that uses the principles of ice-templating to rapidly manufacture polymeric structures into multidirectional 3D architectures with control over pore geometry and size. The fabrication of long-range continuous pore orientation into multi-directions was tested through the control over temperature gradients generated using the 3DICE device. The generated temperature gradients were assessed according to the programmable composition of the 3DICE (e.g. number of plates, sequence of plates, and material composition/conductance of these plates), which governed ice nucleation and growth inside the cryogel. The developed 3D templating strategy presents multiple advantages compared to other macro-scale fabrication methods, namely: (i) costeffective device and method; (ii) small dimensions fabrication system; (iii) fast and miniaturized fabrication approach; (iv) applicable with varied biomaterials; (v) continuous porous structure made of multiple architectures; (vi) 3D coding capabilities; (vii) control from micro- to macro-scale; (viii) fabrication of continuous structures; (ix) results in the spatial control of cell morphology, distribution, and interaction; (x) broad applicability to different cell types, such as stem and endothelial cells.

\section{Materials and methods}

\subsection{Materials}

Gellan gum was purchased from Sigma-Aldrich (Missouri, USA) under the trademark name Gelzan ${ }^{\mathrm{TM}} \mathrm{CM}$. Gelatin from porcine skin gel strength 300, Type A, was purchased from Sigma-Aldrich. Unless otherwise stated, all the reagents were purchased from Sigma-Aldrich.

\subsection{Ice-templating fabrication device}

An acrylic box was designed with $100 \times 30 \times 30 \mathrm{~mm}$ inner height, length, and width. Metal (aluminum) and plastic (PVC) plates with 2 $\mathrm{mm}$ thickness and $100 \times 100 \mathrm{~cm}$ length and width were created, presenting a central hole of $13.5 \mathrm{~mm}$ diameter for the injection of a waterbased gel. Figure S1 shows a representation of the dimensions and configuration of the diverse parts of the device. The custom-made device was then configured with the indicated plates. The installation of plates is adjustable in composition and number. Specifically, for the fabrication of structures with homogeneous linear/vertical porosity, the device was set up with six PVC plates. For the fabrication of homogeneous radial porosity, the device was configured with six aluminum plates. Finally, for the specific case of the random porous structures' fabrication, a Styrofoam plate with $12 \mathrm{~mm}$ height and $100 \mathrm{~mm}$ length and width was placed isolating the central $13.5 \mathrm{~mm}$ mold. In this case, it is important to also isolate the top and bottom of the tube filled with the hydrogel, with two extra layers of Styrofoam, of $2 \mathrm{~mm}$ thickness each.

\subsection{Hydrogel's fabrication}

Gelatin was modified by methacrylation to become photo-sensitive for crosslinking as described elsewhere [29]. Before dissolving both polymers, a solution for visible light crosslinking was prepared adapting the protocol described by Bahney et al., 2011 [30]. Specifically, a solution of distilled water containing $0.75 \%(\mathrm{w} / \mathrm{v})$ of triethanolamine (TEA, Sigma Aldrich) and $37 \mathrm{nM}$ of N-Vinyl-2-pyrrolidone (NVP, Sigma Aldrich) was prepared. Eosin $\mathrm{Y}$ was then dissolved at a concentration of $0.1 \mathrm{mM}$ in the previous solution. To prepare the hydrogel, $2 \%(\mathrm{w} / \mathrm{v})$ GelMA and GG were blended at 4:1 (w:w) in the TEA-VC-Eosin Y solution to produce the GelMA-rich region and the GG-rich region made of GG. The hydrogel was formed inside the ice-templating device by crosslinking triggered by a full spectrum of visible light for $120 \mathrm{~s}$ using a Dentsply Trubyte Triad 2000 (Dentsply Sirona, USA).

\subsection{Random, linear, and radial scaffolds architectures fabrication}

Random scaffold architecture was fabricated using Styrofoam plates, isolating the hydrogel mold all around. The linear architecture was obtained using isolation of the hydrogel with PVC plates all around except on the top surface, where a metal aluminum plain plate was placed to dictate the nucleation of ice crystals. The radial architecture was achieved using isolation on top and bottom surfaces with Styrofoam plates and involving the cylindrical hydrogel mold with the aluminum plates. The device configuration can be observed in Fig. 4. Three different freezing temperatures were applied to test the homogeneous porous scaffold architectures, namely $-20,-80$, and $-196^{\circ} \mathrm{C}$. The hydrogel was directly frozen either with liquid nitrogen $\left(-196^{\circ} \mathrm{C}\right)$, inside the IngClimas $-80{ }^{\circ} \mathrm{C}$ freezer (IngClimas, Barcelona, Spain), or TecnoLar $-20{ }^{\circ} \mathrm{C}$ freezer (TecnoLar, Novo Hamburgo, Brazil). The formed cryogel was then lyophilized using a TELSTAR CryoDos -80 (Azbil Corporation, Japan). The device was never directly placed on the freezer shelf, but on top of a Styrofoam plate inside the freezer, to avoid interference of the shelf temperature if in contact with the bottom part of the device.

When fabricating structures composed by continuous but multidirection pore orientations, namely linear-radial, linear-radial-linear, and radial-linear-radial, alternate plate composition was configured. Specifically:

- Linear-radial: three aluminum plates on top of three PVC plates;

- Linear-radial-linear: two PVC plates on the bottom layer, two aluminum plates in the intermediate layer, and two PVC plated on the top layer;

- Radial-linear-radial: two aluminum plates on the bottom layer, two PVC plated in the intermediate layer, and two aluminum plates on the top layer. 


\subsection{Microtomographic X-rays-computed analysis}

Ice crystals were measured on the obtained dried structure using Xray computed microtomography (micro-CT) analysis in a SKYSCAN 1072 (Bruker, Massachusetts, USA). The structure separation, equivalent to crystal size, was assessed and averaged for the three freezing groups $\left(-196,-80\right.$, and $\left.-20^{\circ} \mathrm{C} ; \mathrm{N}=5\right)$.

Fabricated structures were analyzed by micro-CT after freeze-drying. Images were acquired by X-ray diffraction and then used for a 3D reconstruction. The porosity architecture profile was analyzed, and pore morphometric characterization was performed. 3D projections of the specimens were produced. The 3D structures were acquired with a SkyScan 1272 scanner (v1.1.3, Bruker, Boston, USA), with a pixel size of 10-20 $\mu \mathrm{m}$. Approximately 200-600 projections were acquired over a rotation angle of $360^{\circ}$, with a rotation step of $0.4^{\circ}$. Data sets were reconstructed using standardized cone-beam reconstruction software (NRecon 1.6.10.2, Bruker). The output format for each sample was bitmap images. The representative data set of the slices was segmented into binary images with a dynamic threshold of approximately 20 to 30-255 for the analysis of the polymeric structures (greyscale values optimized per sample and analysis). Then, the binary images were used for morphometric analysis (CT Analyzer, v1.15.4.0, Bruker) and to build the 3D models (CTvox, v 3.0.0, Bruker). When needed, samples were vertically oriented in DataViewer (v1.5.2.3, Bruker) before proceeding to CT Analyzer and CTvox. Pore morphometric analysis was performed in $3 \mathrm{D}$ reconstructed volumes and the following main parameters quantified:

- Pore size distribution: the distribution of pore size frequency, meaning the frequency of pore diameter range. This pore size is calculated based on the pore larger axis (larger diameter).

- Interconnectivity: the interconnectivity was considered as the connectivity in between two or more pores connecting the core of the fabricated structure to the external space. In this particular case, interconnectivity was considered for open pores with more than 40 $\mu \mathrm{m}$ since these structures were tested for biologic assays using human cells approximated to $40 \mu \mathrm{m}$ in diameter.

- Degree of anisotropy: anisotropy is defined as a material's directional dependence of a physical property, being a real number varying from 0 to 1 , from isotropic to anisotropic, respectively. In this case, the directionality of pore and polymeric orientation determines the anisotropic degree. As the number of unidirectional pores increases, the degree of anisotropy increases. Opposingly, isotropy increases as the random distribution of pore orientation increases.

- Pore eccentricity: in mathematics, the eccentricity of a conic section is a non-negative real number that uniquely characterizes its shape, varying from 0 (circular) to 1 (eccentric). In this particular case, eccentricity was quantified on top cross-sections, varying from circular pore shape (eccentricity $=0$ ), to elongated/eccentric pore shape (eccentricity $=1$ ).

- Polymeric number: the polymeric number represents the number of polymeric structures (pore walls) per unit of space (millimeter). Usually, as pore size decreases, the polymeric number per millimeter increases.

\subsection{Structures pore orientation analysis using FIJI}

Images collected with micro-CT, namely cross-sections of random, linear, radial structures, and combinations thereof, were used in FIJI [31]. First, greyscale images were converted into binary format. Then, the Process tool "Subtracting background" was applied to all images. To quantify pore orientation frequency, the Analyze tool "Directionality" was applied, from -90 to $90^{\circ}$ and the Local Gradient Orientation method used $(\mathrm{N}=6$, deviation demonstrated as a confidence interval of 95\%). When combinations of Linear-Radial, Linear-Radial-Linear, and
Radial-Linear-Radial architectures were analyzed, three sections per structure were independently created and quantified $(\mathrm{N}=3$, deviation demonstrated as a confidence interval of $95 \%)$.

\subsection{Dynamic mechanic analysis}

The viscoelastic measurements were performed using a TRITEC8000B dynamic mechanical analyzer (Triton Technology, UK) in the compressive mode. Samples were cut into cylindrical shapes of approximately $3 \mathrm{~mm}$ diameter and $3 \mathrm{~mm}$ thickness and immersed in phosphate buffer solution (PBS) until equilibrium was reached $\left(37^{\circ} \mathrm{C}\right.$, overnight). After equilibration at $37^{\circ} \mathrm{C}$, the DMA spectra were obtained during a frequency scan between 0.1 and $10 \mathrm{~Hz}$. The experiments were performed under a constant displacement amplitude $(50 \mu \mathrm{m})$, always at $37{ }^{\circ}$ C. A small preload $(0.005 \mathrm{~N})$ was applied to each sample to ensure that the entire structure surface was in contact with the compression plates before testing, and the distance between plates was equal for all structures being tested $(n=4)$.

\subsection{Scanning electron microscopy (SEM)}

The structures were analyzed by SEM (NanoSEM-FEI Nova 200, USA) to assess their micro/nanoarchitecture. Prior to the microstructure imaging by SEM, specimens were coated with gold using a Fisons Instruments Coater (Quorum/Polaron E6700, UK) with a current set at 18 $\mathrm{mA}$, for a coating time of $120 \mathrm{~s}$. SEM was performed to evaluate architecture morphology.

\subsection{Cell isolation and culture}

Fat pad-ASCs were isolated from human tissues obtained from Hoffa's body removed during arthroscopic surgeries on male and female donors with ages between 19 and 32 years, after informed consent, under cooperation protocols established with Centro Hospitalar Póvoa do Varzim, and Clínica Saúde-Atlântica. The study has been approved by the Ethical Committees of Centro Hospitalar Póvoa do Varzim, Grupo Saúde-Atlântica and the University of Minho.

All the samples were processed within $24 \mathrm{~h}$ after the arthroscopic procedure to the knee. hASCs were isolated following an enzymatic digestion-based method with type II collagenase (Sigma) and were characterized by flow cytometry (figure S3). The extracted tissue was placed in PBS solution and washed several times with PBS containing 1\% (v/v) antibiotic/antimycotic (AB) mixture, until total removal of blood and cut into small pieces. Tissue digestion was performed by incubation at $37{ }^{\circ} \mathrm{C}$ in a humidified atmosphere of $5 \% \mathrm{CO}_{2}$ for $10 \mathrm{~h}$ in a $10-20 \mathrm{~mL}$ 1:1 mixture of Minimum Essential Media alpha (MEM alpha, Invitrogen) supplemented with $10 \%$ fetal bovine serum (FBS, Gibco) and $1 \% \mathrm{AB}$ mixture, with type II collagenase $0.15 \%(w / v)$ in PBS. The digested tissue was filtered, and the obtained cell suspension was centrifuged at $300 \mathrm{~g}$ for $5 \mathrm{~min}$. The hASCs were selected by plastic adherence and expanded in $\alpha$-MEM medium supplemented with $10 \%$ FBS and $1 \%$ of AB mixture.

Human Hoffa's body isolated ASCs were seeded in a concentration of $5 \times 10^{6}$ cells per scaffold, half on its top and half on its bottom surfaces, and cultured for 21 days in the different architectures using a 24-well suspension culture plate (VWR). When co-cultured with GFPexpressing hUVECs (Lonza, Switzerland), the total amount of 5 million cells were kept using a ratio of 1:4 ASCs:hUVECs. Cells were cultured with a combination of 1:4 hepatic medium and EGM-2 (Lonza), respectively. The fraction of hepatic induction medium was DMEM lowglucose (Sigma) supplemented with $20 \mathrm{ng} / \mathrm{mL} \mathrm{HGF}$ (Sigma) and $10 \mathrm{ng} /$ mL bFGF (PeproTech, UK), nicotinamide $0.61 \mathrm{~g} / \mathrm{L}$ (Sigma), for 7 days (induction period), followed by the maturation medium, consisting of DMEM low-glucose supplemented with $20 \mathrm{ng} / \mathrm{mL}$ oncostatin M (Sigma), $1 \mathrm{~mol} / \mathrm{L}$ dexamethasone (Sigma), and $50 \mathrm{mg} / \mathrm{mL}$ Insulin-TransferrinSelenium (ITS, Alfagene, Portugal), $1 \% \mathrm{AB}$, for more 14 days 
(maturation period). Medium changes were performed twice a week. On day 21, cell culture was ended to perform immunocytochemical analysis. Each architecture formulation was compared against each other.

\subsection{Immunostaining and quantification}

ASCs (red) were live stained using CellTrackerTM CM-DiI Dye (Thermo-Fisher). Samples from the in vitro assays were fixed with $10 \%$ formalin for $20 \mathrm{~min}$ at room temperature and washed with PBS. The blue fluorescent 4',6-diamidino-2-phenylindole (DAPI) was used at $0.02 \mathrm{mg}$ / $\mathrm{mL}$ final concentration, $1: 1000(\mathrm{v} / \mathrm{v})$ dilution, as a nuclear counterstaining. After washing, 3D constructs were visualized with a transmitted and reflected light microscope (Axio Imager Z1m, ZEISS). AntiOsteopontin antibody (ab8448) was used for OPN detection. The secondary antibody Alexa Fluor 594 Donkey Anti-Rabbit IgG $(\mathrm{H}+\mathrm{L})$ (Thermo Fisher, USA) was used to label the biomarker fluorescently. hUVECs were expressing GFP (Lonza) to track endothelial segments formation over 21 days.

\subsection{Confocal microscopy}

Images were acquired using an inverted confocal laser scanning microscope TCS SP8 (Leica Microsystems, Wetzlar, Germany). Fluorescently stained cells cultured in the random, linear, and radial scaffolds architecture were assessed by confocal microscopy and 3D reconstructed (LAS X software, Leica). The samples were observed by using fluorescence $\lambda_{\mathrm{ex}}=358 \mathrm{~nm} \lambda_{\mathrm{em}} 461 \mathrm{~nm}$ for nuclei stained by DAPI, and $\lambda \mathrm{ex}=540 \mathrm{~nm} \lambda \mathrm{em}=565 \mathrm{~nm}$ for rhodamine phalloidin stained $\mathrm{F}$ actin.

\subsection{CYP450 activity quantification and cell morphological analysis}

P450-Glo ${ }^{\mathrm{TM}}$ Assay (Promega, Wisconsin, USA) method was applied to measure cytochrome P450 (CYP) activity. A CYP enzyme and a P450$\mathrm{Glo}^{\mathrm{TM}}$ substrate were combined in potassium phosphate (KPO4) buffer with or without a test compound of interest, and the reaction was initiated by adding an NADPH regenerating system, following the instructions provided by the supplier. $25 \mu \mathrm{l}$ of the mixture was combined with an equal volume of test compound solution to give one-half of the final reaction volume in a 96 -well plate. The reaction was initiated by adding two volumes of $2 \mathrm{X}$ concentrated NADPH Regeneration System. Then, an equal volume of Luciferin Detection Reagent was added to simultaneously stop the CYP reaction and initiate a luminescent signal that is proportional to the amount of CYP activity. Signal was allowed to stabilize for about $20 \mathrm{~min}$ at room temperature before reading luminescence on a luminometer microplate reader (Synergy HT, BioTek Instruments, USA). The blank control was performed on scaffolds without cells incubated with the same reaction mix. The concentration of dluciferin generated by CYP in P450-Glo ${ }^{\mathrm{TM}}$ Assays was determined by comparing luminescence from CYP reactions to luminescence from a dluciferin standard curve. By comparing signals from CYP reactions to those from d-luciferin standards, the P450 activity was quantified ( $\mathrm{N}=$ $6)$.

While cell orientation was assessed using the FIJI tool "Directionality", cell morphology was analyzed using the tool "Analyze Particles". Microscopic images were converted into binary images, despeckled, bright and dark outliers up to 5 pixels were removed. The cell body was quantified between 300 and $3000 \mu \mathrm{m}^{2}$ to avoid artifacts and aggregated cells, while nuclei were identified between 30 and $300 \mu \mathrm{m}^{2}$. Objects touching the image borders were excluded. The endothelial network, more specifically the major segments, branches, and meshes, were identified and quantified with the FIJI plugin Angiogenesis Analyzer. The previously described image treatment was applied to greyscale images for endothelial network identification. Confocal images were obtained, and 3D reconstructed. Different thin slices along the constructs were used for imaging and quantification.

\subsection{Statistical analysis}

The investigated parameters were expressed as mean \pm standard deviation, confidence interval, or standard error of the mean. Not-paired two-tailed Student's t-tests, One-way, and Two-way analysis of variance (ANOVA) with Tukey test, were performed to determine the significance of the results, and a p-level $<0.05$ was considered statistically significant. Statistical analyses were carried out with Microsoft Excel 2016 and GraphPad Prism 9 for Windows.

\section{Results}

\subsection{Device development for controlled ice nucleation and growth}

To precisely control the ice crystal growth orientation in freezing gels, we developed the 3DICE, a system to thermally fabricate 3D porous architectures in cryogels. The 3DICE (Fig. 1A-i) consists of an acrylic box where series of metal or plastic layers, or both, sit in (Fig. 1A-ii). The aluminum metal plates, $237 \mathrm{~W} /(\mathrm{m} \cdot \mathrm{K})$ thermal conductivity, and the polyvinyl chloride (PVC) plastic ones, of thermal conductivity ranging from 0.14 to $0.28 \mathrm{~W} / \mathrm{m} . \mathrm{k}$, of custom positioning in the device, were idealized to test the hypothesis of controlling the ice crystals growth in specific orientations based on the different thermal conductivity existing in contact with a water-based gel (Fig. 1A-iii). The thermal conductivity of each plate controls the ice crystal growth orientation radially or vertically/linearly during the gel freezing. When a region is isolated by a low thermal conductivity plate, the ice crystals grow vertically. When a region of the gel is surrounded by a highly thermal conductive metal plate, the ice crystals grow radially towards the plate.

To assess the ice-crystal formation process in hydrogels crosslinked by visible light and calcium ions, using the developed system, blended methacrylated gelatin (GelMA) and low acyl gellan gum (GG) were injected in the central hole of the 3DICE when fully surrounded by Styrofoam before freezing. Pure GG was also tested, proving the versatility of the system for different gel polymeric compositions (figure S2). Temperatures of $-20{ }^{\circ} \mathrm{C},-80^{\circ} \mathrm{C}$, and $-196{ }^{\circ} \mathrm{C}$ were applied and the geometry of ice nucleation was qualitatively assessed (Fig. 1B-i). The resulting porous structures were characterized by a decreasing total porosity and interconnectivity with the decrease of freezing temperature (Fig. 1B-ii). While the bi-dimensional pore shape eccentricity was not influenced by the processing temperature (Fig. 1B-iii), the pore size significantly decreased as the freezing temperature was lowered $(\mathrm{p}<$ 0.05; Fig. 1B-iv). Still, regarding the structure shape, the structure model index indicated that when applying a $-20{ }^{\circ} \mathrm{C}$ freezing step, the surface of polymeric structures was significantly characterized by planar and rod/cylindrical geometries, while at $-80^{\circ} \mathrm{C}$ it was mainly composed by planar plates, and when using $-196{ }^{\circ} \mathrm{C}$ freezing temperature, the obtained inner polymeric surfaces were concave (Fig. 1B-v). The fractal dimension also corroborates that the shape of the inner structure was mainly composed of planar plates, which tended to become more complex when frozen at lower temperatures. The number of polymeric structures composing the whole fabricated structure, and its connectivity, increased as the applied freezing temperature decreased (Fig. 1B-vi). All these geometrical parameters were directly influenced by the formed ice-crystals during the freezing step of the hydrogel conversion into cryogel, which directly varied with the temperature (Fig. 1B-vii). Additionally, a linear regression was performed to fit the ice crystal size varying with the controlled freezing temperatures. The obtained regression equation is: $y=0.0015 x+0.3575$. The units of the variables are millimeter for $y$, and Celsius degree for $x$. The fit had a resulting $R$ value of 0.997 . A positive $R$ value indicates that as the freezing temperature increases the ice crystal size also increases, and an $\mathrm{R}$ close to 1 shows that knowing the value of the first variable allows perfect prediction of the second one. 

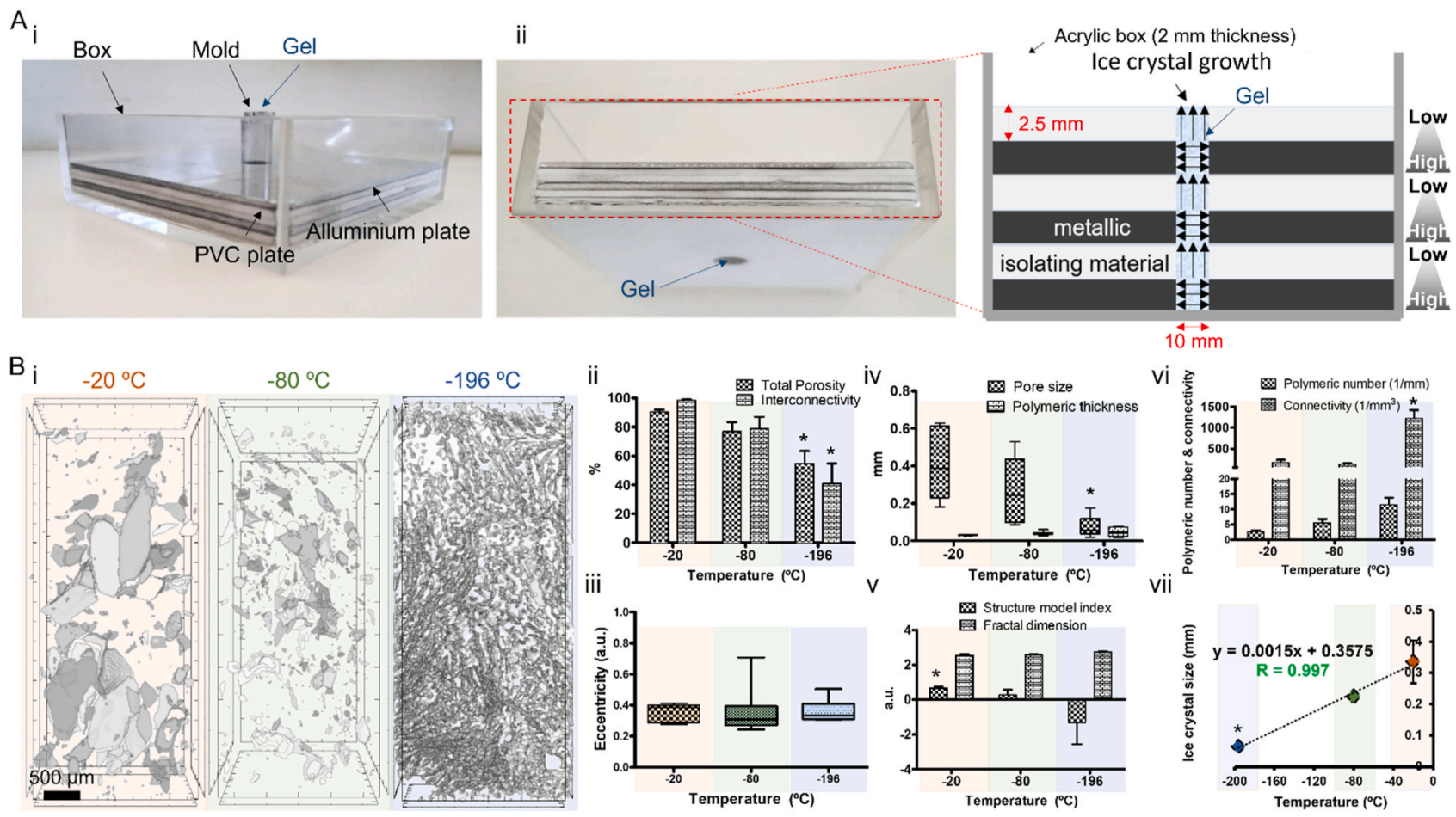

Fig. 1. Developed device for controlled ice-templating and 3D pore geometry. (A) The 3DICE composed of sequences of metal and plastic-based plates of different thermal conductivities was fabricated to test the hypothesis of controlling ice crystals morphology and guide its growth in cryogels. Photography of the device demonstrates (i) how different plates are inserted inside a mold with a central hole for gel injection (scale bar $=1 \mathrm{~cm}$ ). (ii) A lateral view of the $3 \mathrm{DICE}$ demonstrates the thickness $(2.5 \mathrm{~mm}$ ) and a series of plates, which can be easily adapted and customized (scale bar $=1 \mathrm{~cm}$ ). (iii) The lateral representation of a crosssection of the 3DICE demonstrates the specific dimensions and customizable set of plates. According to our hypothesis, the schematic shows the hypothesis of the ice crystal formation guidance (i.e. radially by the metal plates and vertically by the plastic plates). (B) A first test performed with a gel fully covered by Styrofoam to induce random ice crystal formation at the three different temperatures was performed. The 3D reconstruction and assessment of the formed porosity induced by random ice crystal formation at $-20,-80$, and $-196^{\circ} \mathrm{C}$ (i) showed the crystal distribution and shape in the $3 \mathrm{D}$ hydrogel space (scale bar $=500 \mu \mathrm{m}$ ). The applied temperatures of $-20^{\circ} \mathrm{C},-80^{\circ} \mathrm{C}$, and $-196^{\circ} \mathrm{C}$ controlled the (ii) amount of total porosity and interconnective pores (for cell biology applications we have considered $>40 \mu \mathrm{m}$ interconnective space diameter in between pores), (iii) ice crystal mean 2D eccentricity, (iv) pore size and polymeric wall thickness, (v) 3D pore shape indexes - structure model index and fractal dimension, (vi) the number of polymeric walls and connective pores per unit of space, and (vii) the average ice crystal size formed at the three different temperatures during the freezing step. A linear regression was used to fit the ice crystal size dependence on freezing temperature. The regression equation and its $\mathrm{R}$ value are indicated. Data shown as Mean $\pm \mathrm{SD}, \mathrm{N}=6$, * $\mathrm{p}$-value $<0.05$.

\subsection{Tuning pore geometry and size in $3 D$ random, linear, and radial orientations}

Three specific architectures of 3D porous structures were tuned by the 3DICE composition in metal or plastic molding plates (Fig. 2). After freeze-drying, random, linear, and radial scaffolds (classification according to the longitudinal section) were produced when Styrofoam, plastic plates, and metal plates were applied, respectively. Although radial orientation was observed from the top view, the formed porosity in the "linear" configuration resulted in pores vertically interconnected through the scaffold (longitudinal section), while the formed porosity in the "radial" configuration resulted in parallel radial plates through the structure (longitudinal section). In more detail, while Styrofoam was used to produce random isotropic porous structures (Fig. 2A-i), multiple PVC or aluminum plates were applied to linearly/vertically (Fig. 2A-ii) or radially (Fig. 2A-iii) guide the pore formation through the longitudinal section of the resulting structures, respectively.

To control the pore size distribution of the fabricated 3D structures, the strategies described above were processed under $-20^{\circ} \mathrm{C},-80{ }^{\circ} \mathrm{C}$, and $-196{ }^{\circ} \mathrm{C}$. The mean pore size tended to decrease as the freezing temperature decreased, by tuning the ice crystal nucleation. At $-20{ }^{\circ} \mathrm{C}$, the mean pore sizes of random, linear, and radial architectures were
407,329 , and $335 \mu \mathrm{m}$, while freezing at $-80^{\circ} \mathrm{C}$ resulted in 273,263 , and $224 \mu \mathrm{m}$, respectively. When the lowest temperature was reached, $-196^{\circ} \mathrm{C}$ of liquid nitrogen, the observed mean pore sizes were 75,80 , and $64 \mu \mathrm{m}$, respectively.

\subsection{Anisotropy, translucency, and mechanical response of random, linear, and radial architectures}

To compare the obtained architectures, using X-ray computed microtomography (micro-CT), the degree of anisotropy was quantified for the applied freezing temperatures. Generally, linear $\left(-20^{\circ} \mathrm{C}=0.5\right.$; $\left.-80{ }^{\circ} \mathrm{C}=0.6 ;-196{ }^{\circ} \mathrm{C}=0.5\right)$ and radial architectures $\left(-20{ }^{\circ} \mathrm{C}=0.4\right.$; $-80{ }^{\circ} \mathrm{C}=0.6 ;-196{ }^{\circ} \mathrm{C}=0.4$ ) presented higher degree of anisotropy compared to the random one obtained through isotropic freezing $\left(-20{ }^{\circ} \mathrm{C}=0.4 ;-80{ }^{\circ} \mathrm{C}=0.4 ;-196{ }^{\circ} \mathrm{C}=0.3\right.$; Fig. 3A-i). The specific architectures maintained similar porous arrangements, but reduced pore size as the freezing temperature decreased (Fig. 3A-ii). The 2D geometry of the pores was calculated as more elongated at -20 and $-80{ }^{\circ} \mathrm{C}$ (Fig. 3A-iii), once the number of polymeric walls was lower per unit of space when compared to $-196^{\circ} \mathrm{C}$ (Fig. 3A-vi).

To test the dependence between translucency and dynamic mechanical response with structure architecture, visible light spectroscopy, 
A i

Random ii

Linear

iii

Radial

Top view Longitudinal view
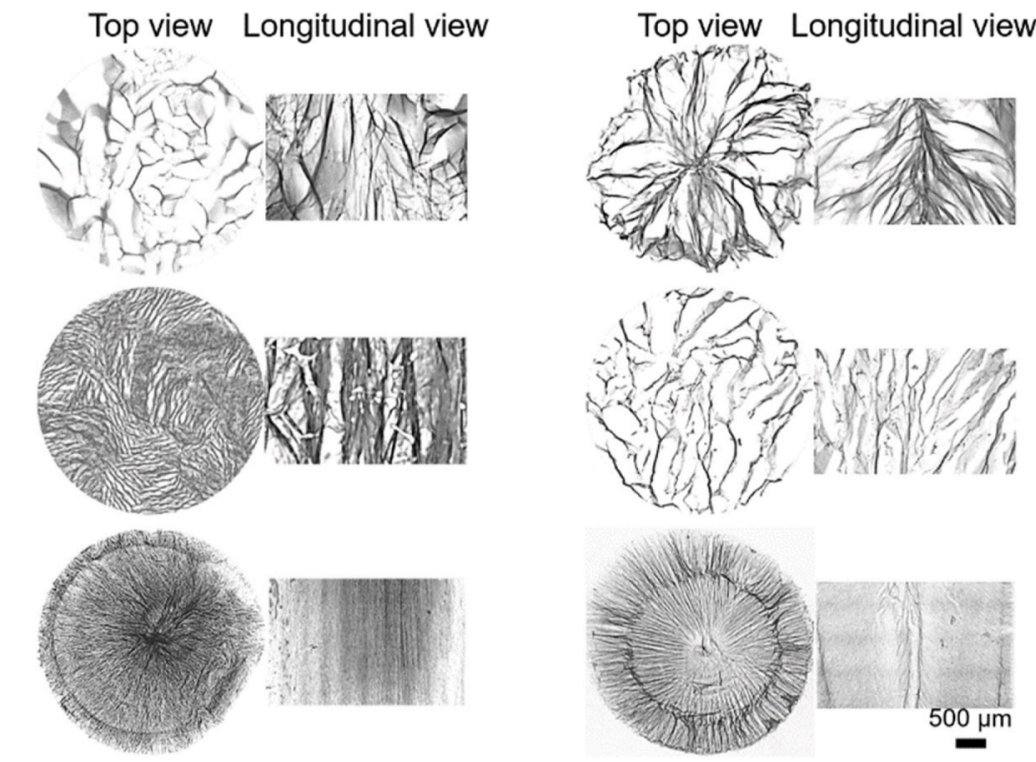

$-20^{\circ} \mathrm{C}$

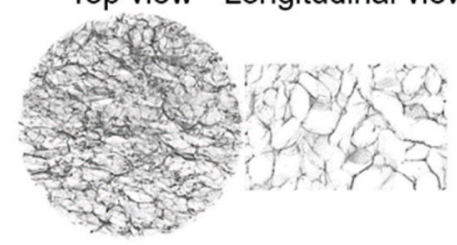

B i

ii

Linear

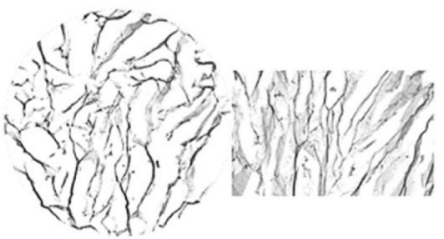

$-196^{\circ} \mathrm{C}$

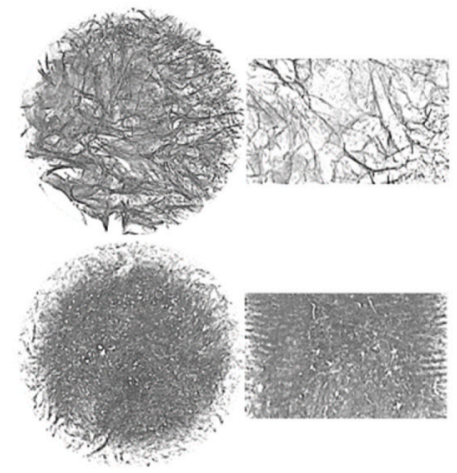

Random
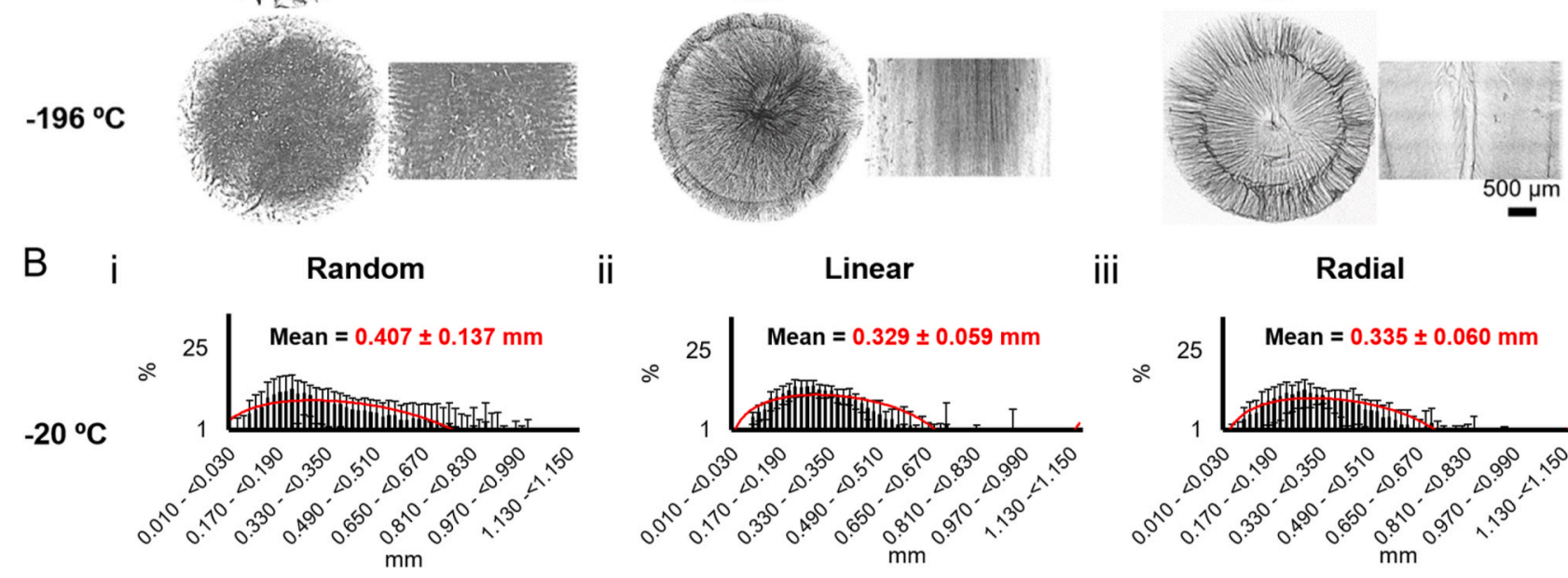

iii

Radial
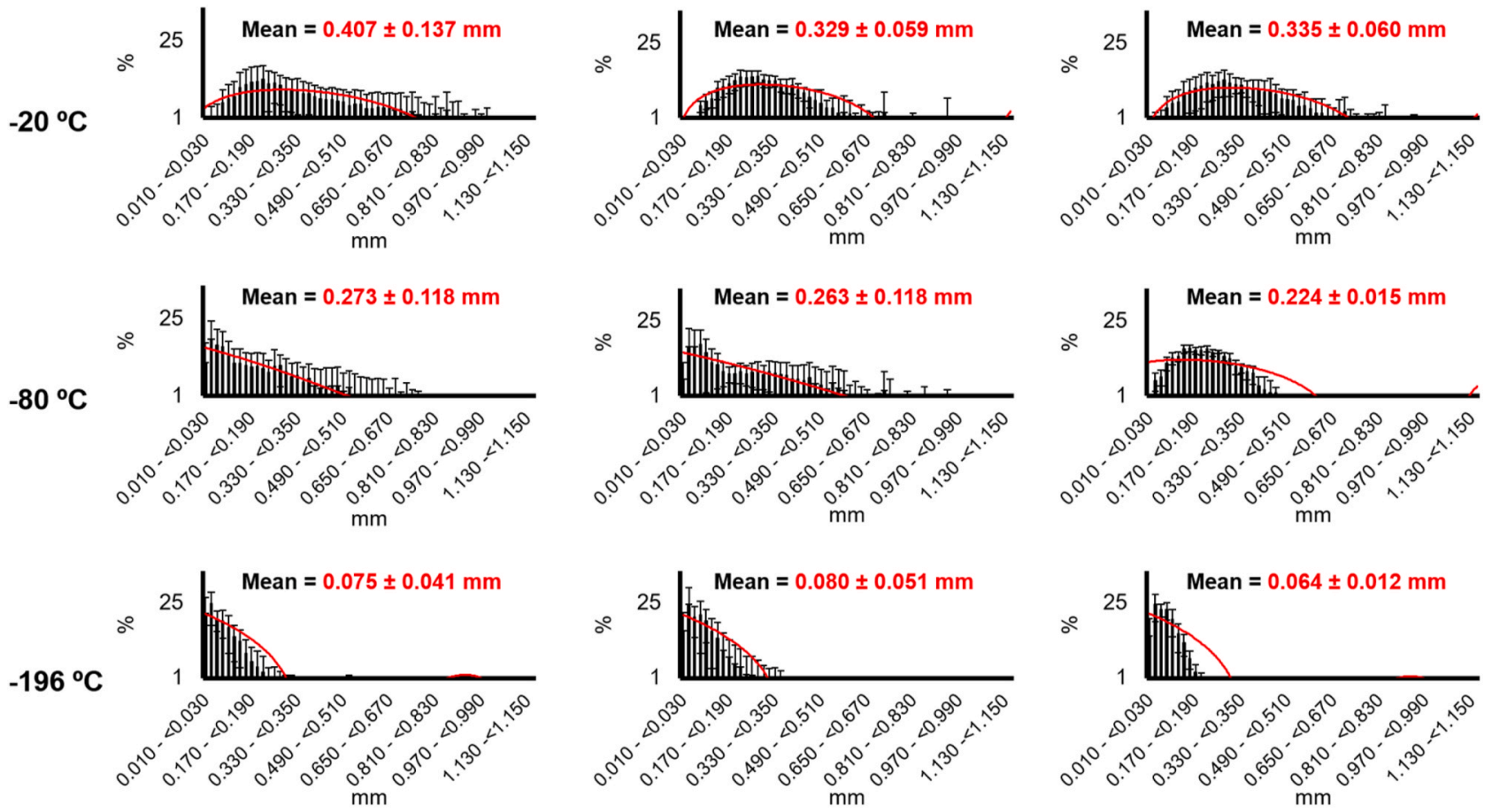

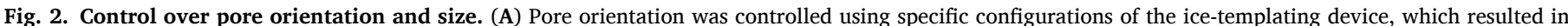

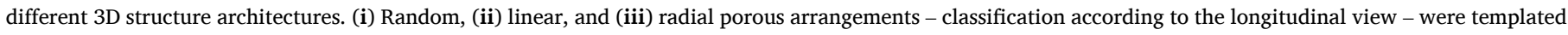

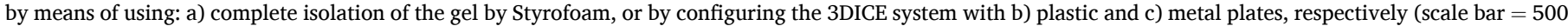

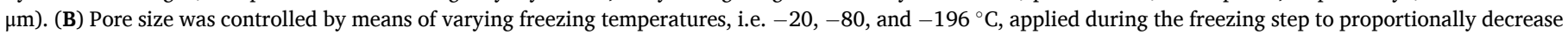
the pore size. Data are shown as Mean $\pm S D(N=6)$.

and DMA were performed, keeping the polymeric composition and concentration constant (Fig. 3B-i). Radial porous architecture resulted in increased light transmittance. Similarly, larger pores led to increased light transmittance, enhancing structure translucency. When a dynamic force was applied on the top of the structures at increasing frequencies, the storage modulus of the linear architectures was higher than of the random and radial ones (Fig. 3B-iii). On the other hand, the $\tan \delta$ reaction to dynamic compression at higher frequencies in random and radial architectures was higher than in the linear one (Fig. 3B-iv). Pore arrangement was additionally demonstrated by magnified imaging obtained through SEM, showing the random, linear, and radial microscopic architectures (Fig. 3B-v). 
A

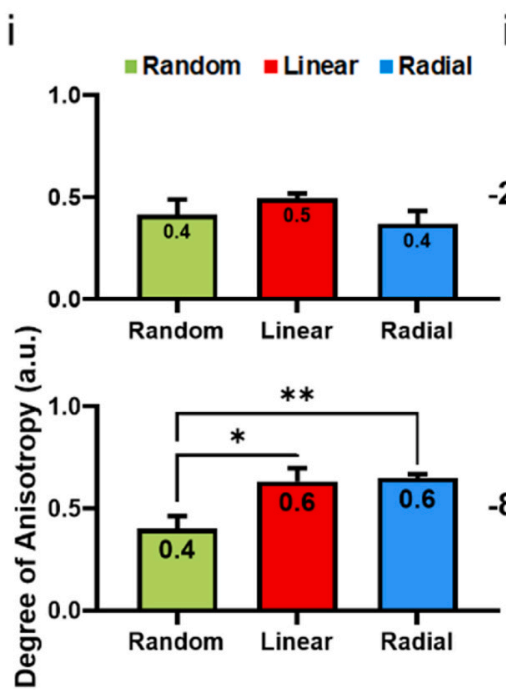

ii

$20^{\circ} \mathrm{C}$

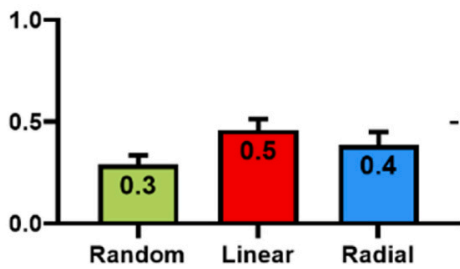

$-80^{\circ} \mathrm{C}$

$\mathrm{Bi}$

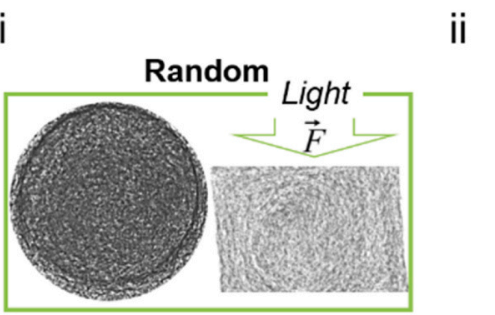

$196{ }^{\circ} \mathrm{C}$
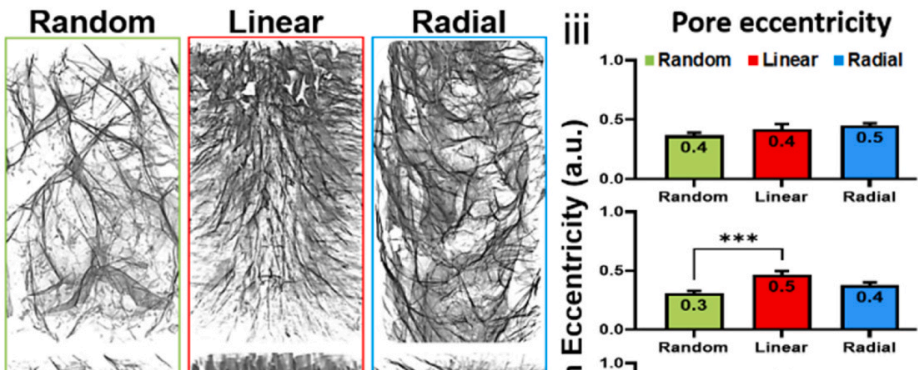

กิ
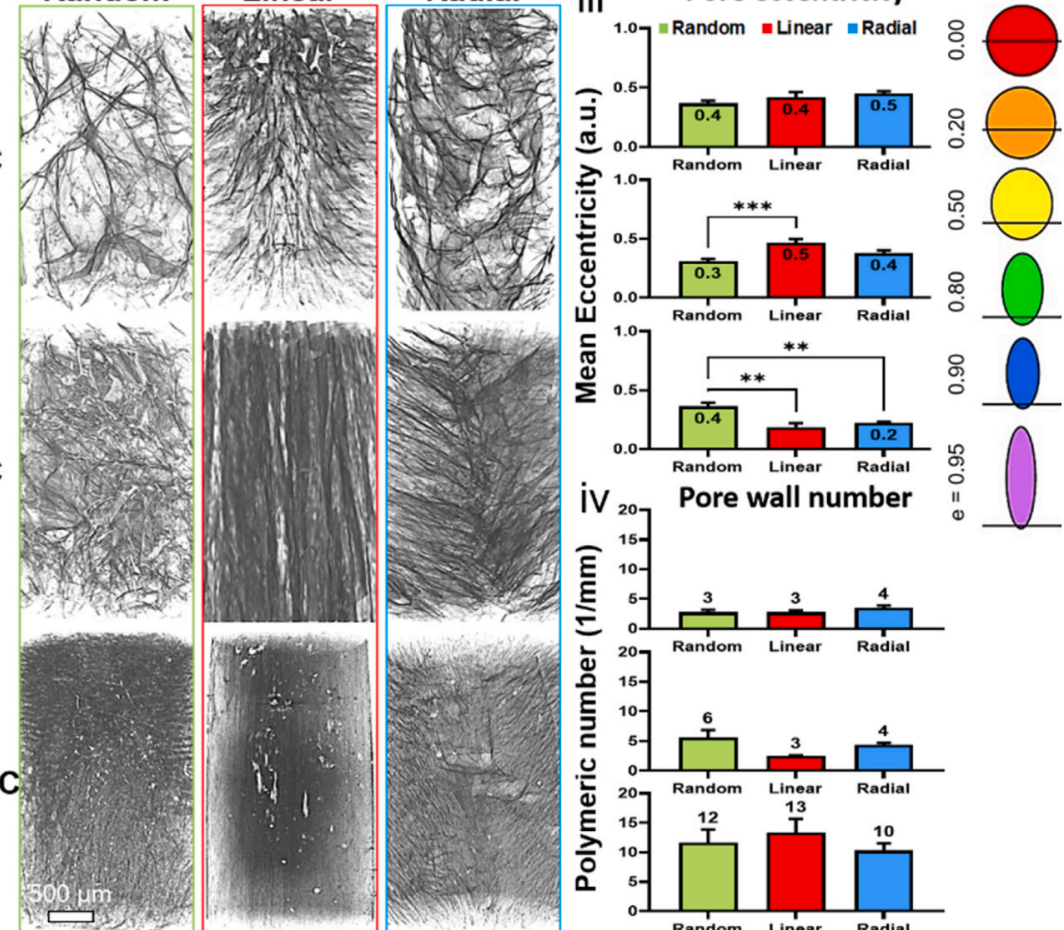

웅

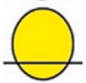

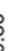

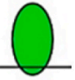

.

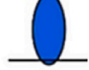

iv $^{20}$ Pore wall number
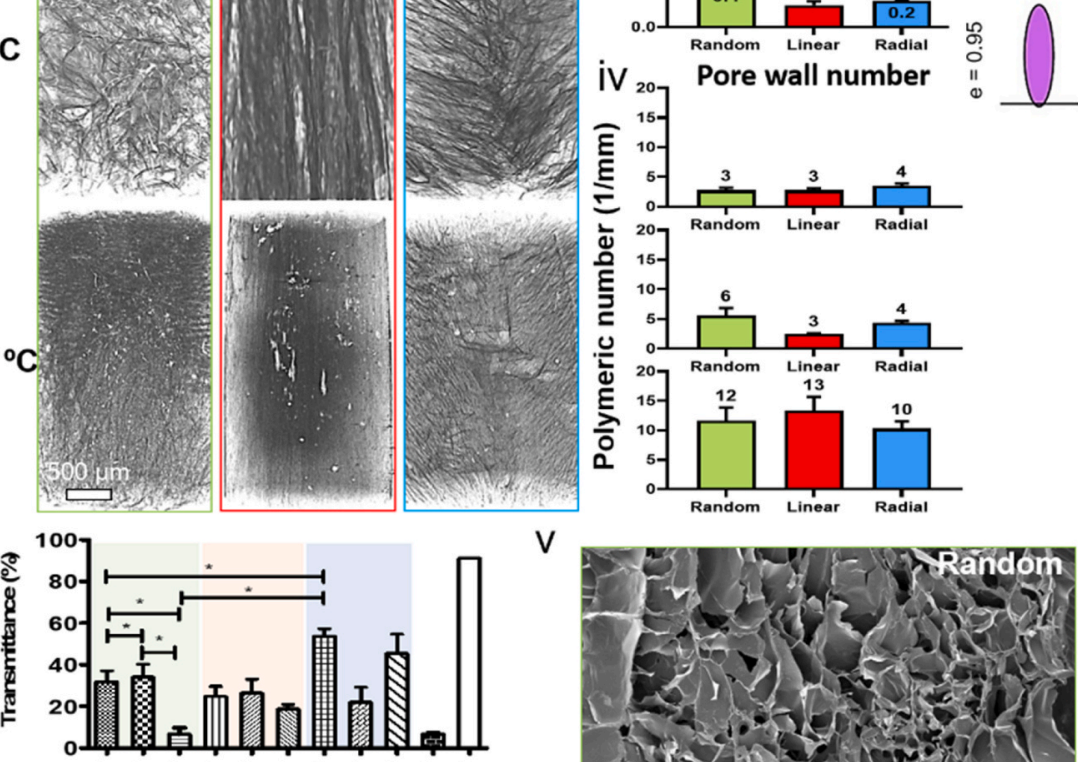

V
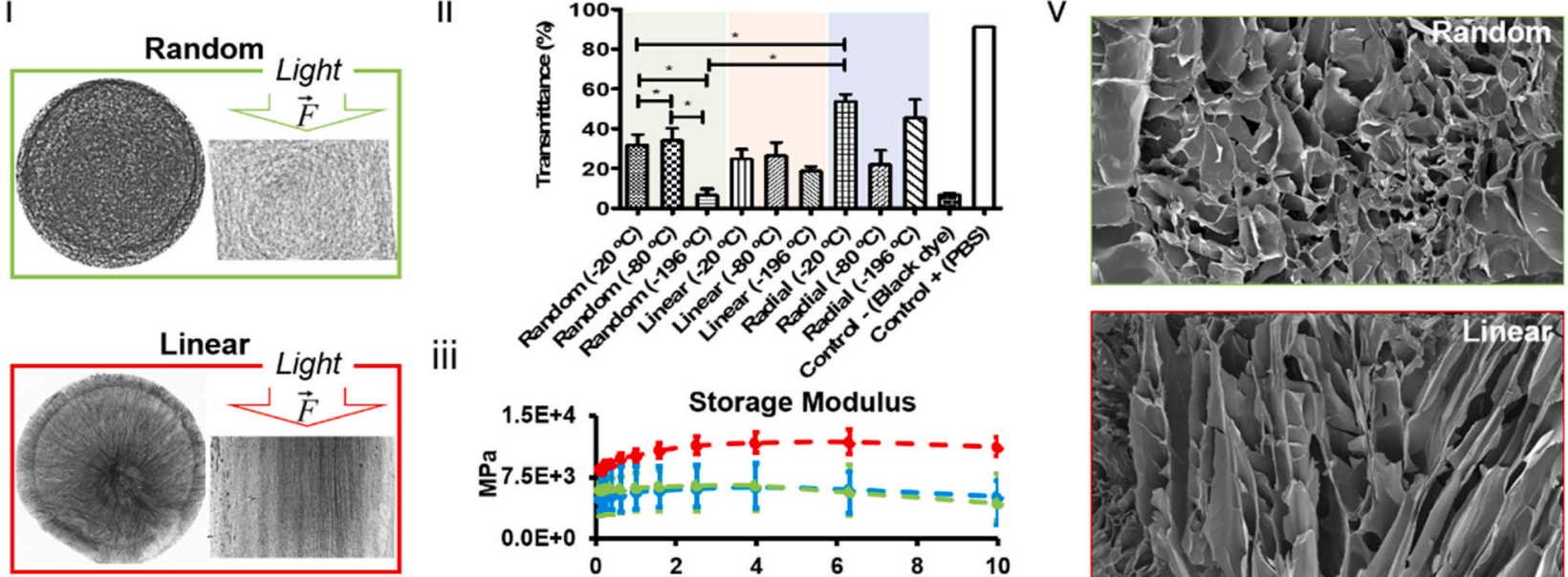

iii
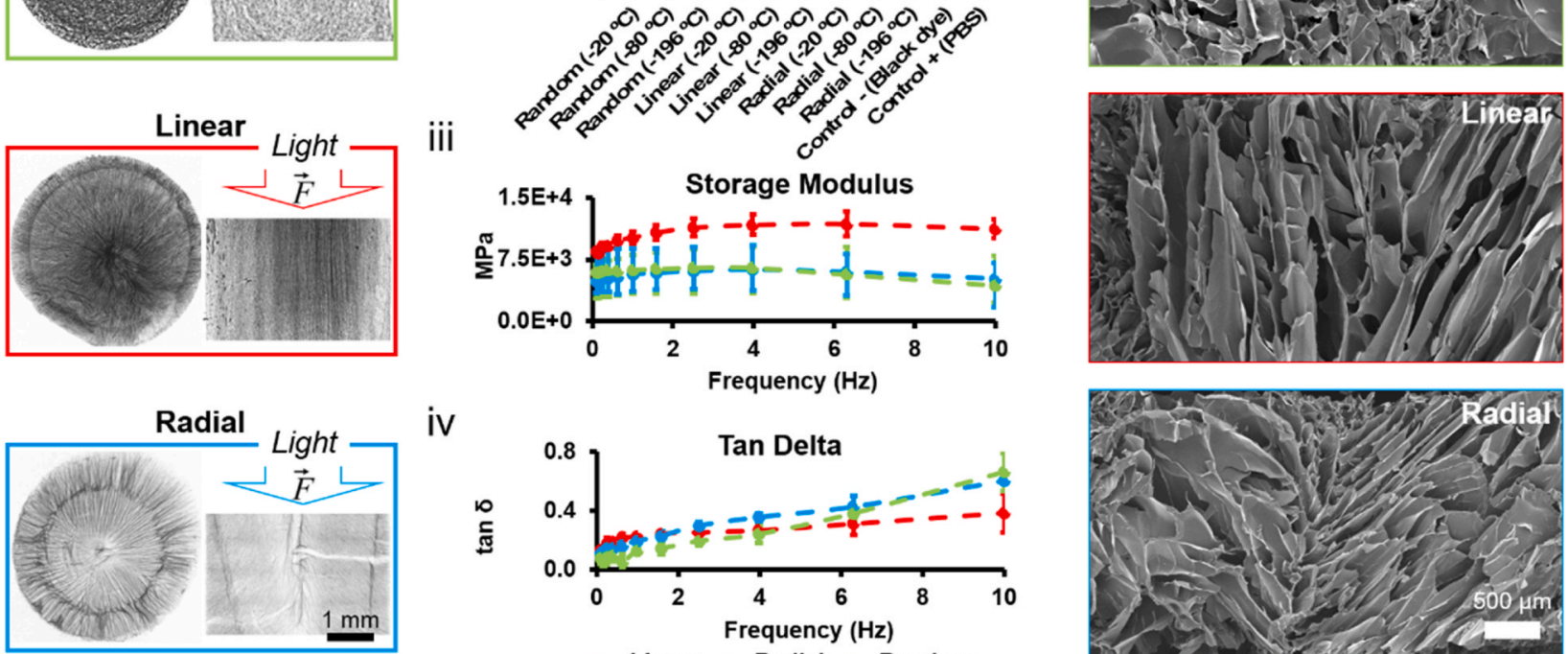

iv
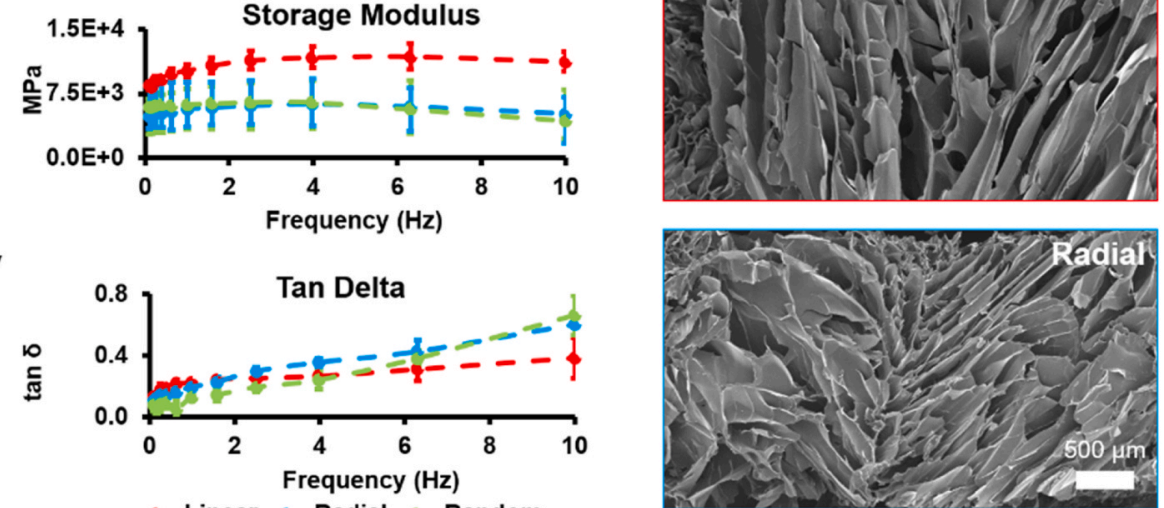

Fig. 3. Correlation between the degree of anisotropy and mechanical response of 3D porous scaffolds. (A) The degree of anisotropy (i) of random, linear, and radial architectures (ii) was compared for $-20,-80$, and $-196^{\circ} \mathrm{C}$. (iii) $2 \mathrm{D}$ mean eccentricity was quantified on cross-sections from the top. (iv) Polymeric number assessed through lateral cross-section of the different architectures $(\mathrm{N}=9-11)$. (B) The three architectures fabricated at $-196{ }^{\circ} \mathrm{C}$ were analyzed for (i) light transmittance and dynamic mechanical response $(\mathrm{N}=4)$ after immersion in PBS. (ii) Light transmittance was plotted against porosity architecture to assess translucency $(\mathrm{N}=4)$. (iii) The Storage Modulus and (iv) Tan Delta $(\delta)$ response were quantified for increasing top compression frequencies ranging from 1 to $10 \mathrm{~Hz}$. (v) Scanning electron microscopy (SEM) micrographs showing the pore arrangement. Data shown as Mean \pm SEM (*p-value $<0.05$; **p-value $<0.005$; ***p-value $<0.001)$.

\subsection{Coding pore orientation into multidirectional 3D architectures}

In Fig. 4, different schemes for controlling the formation of random, linear, and radial porosities are shown (Fig. 4A-i). So, to code specific architectures in cryo-gels, GelMA-GG gels were injected into the mold and crosslinked using visible-light. While Styrofoam was used to produce (ii) random porosity, compiled PVC or aluminum plates were used to generate (iii) linear, or (iv) radial porosities, respectively. 

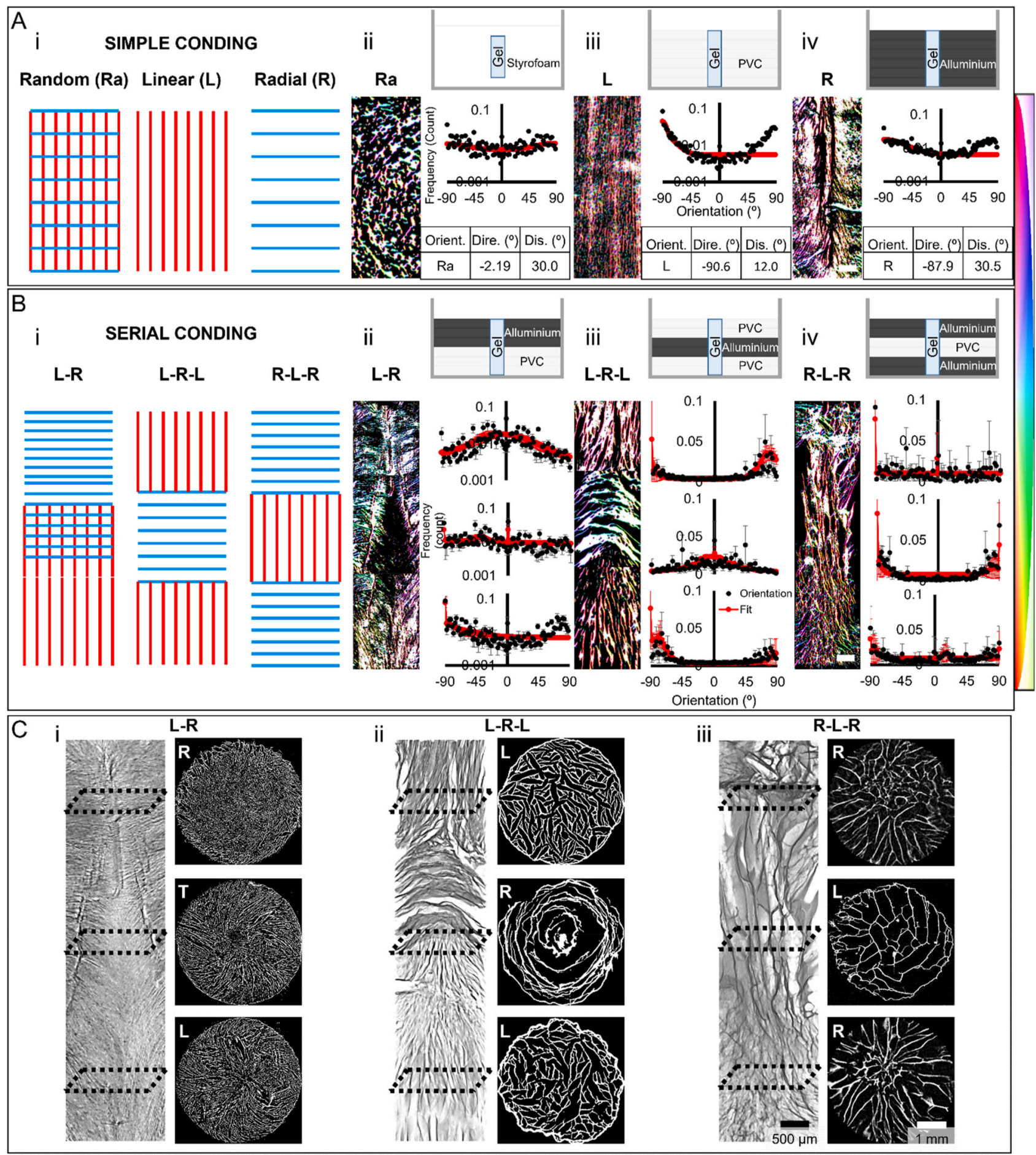

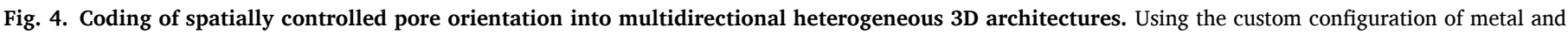

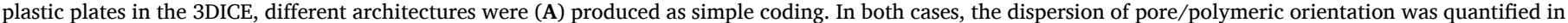

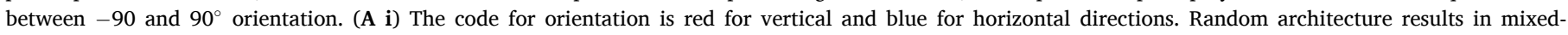

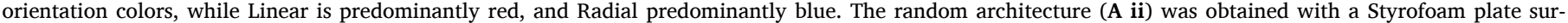

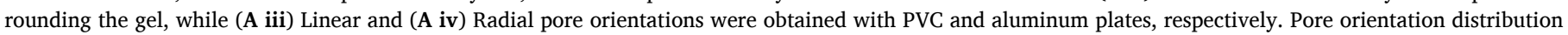

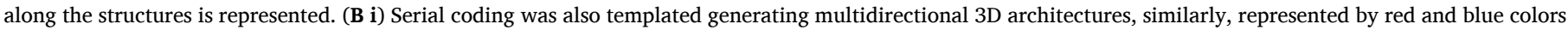

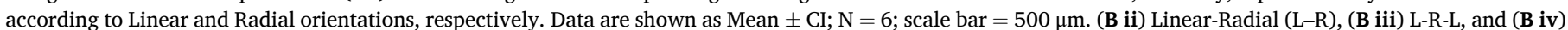

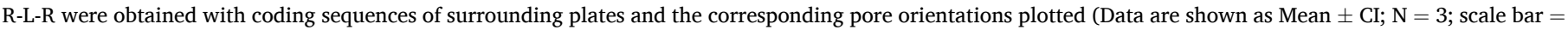

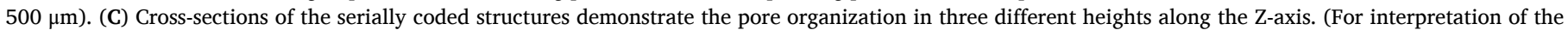
references to colour in this figure legend, the reader is referred to the Web version of this article.) 
To generate complex multidirectional 3D pore architectures, specific configurations of intercalated metal and plastic plates were inserted into the 3DICE before gel injection and crosslinking to produce specific sequences of pore orientation (Fig. 4B-i). These results demonstrated the control over the orientation of porosity along the Z-axis. Sequences of (ii) Linear-Radial, (iii) Linear-Radial-Linear, and (iv) Radial-Linear-
Radial were verified.

Overall, when pore orientation was predominantly vertical, the directionality frequency graphs showed two peaks at -90 and $90^{\circ}$, as observed in Fig. 4-A and -B, ii), iii), and iv). Opposingly, when the pore orientation was predominantly radial, directionality graphs were represented by one peak at $0^{\circ}$, or two peaks between 0 and $45^{\circ}$. Vertical
A

B

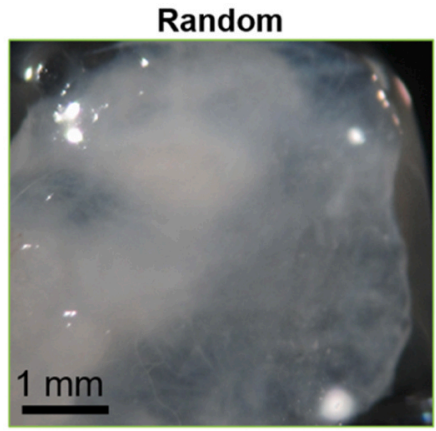

i
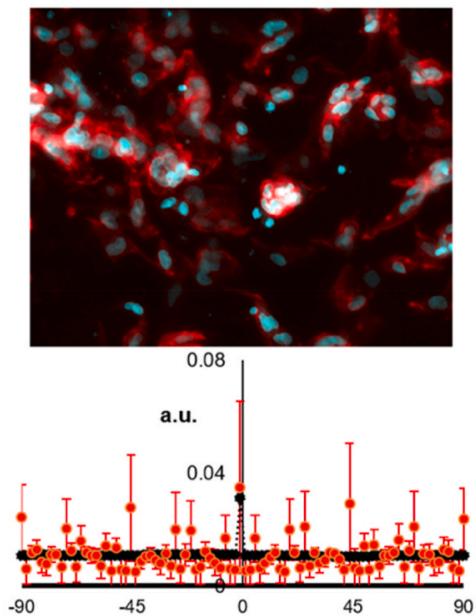

Linear

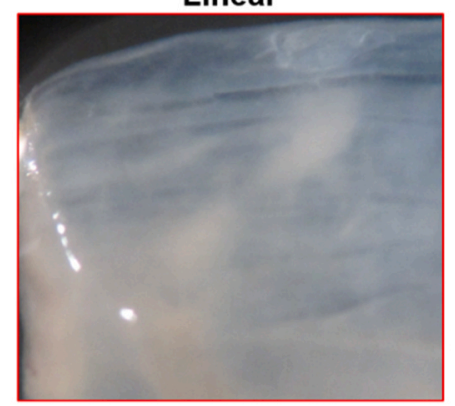

ii
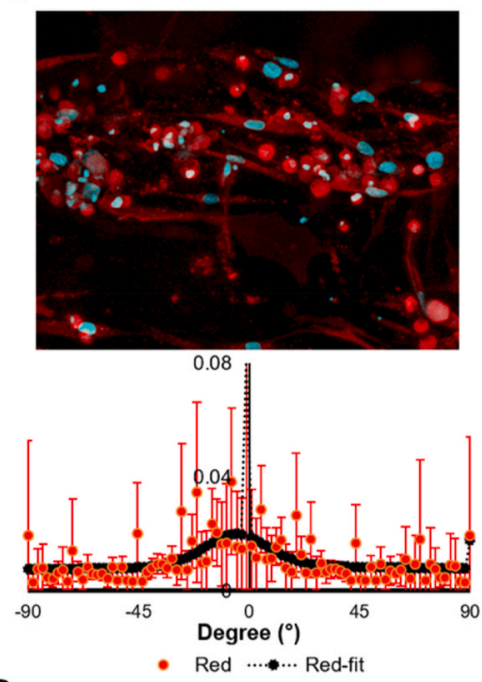

D
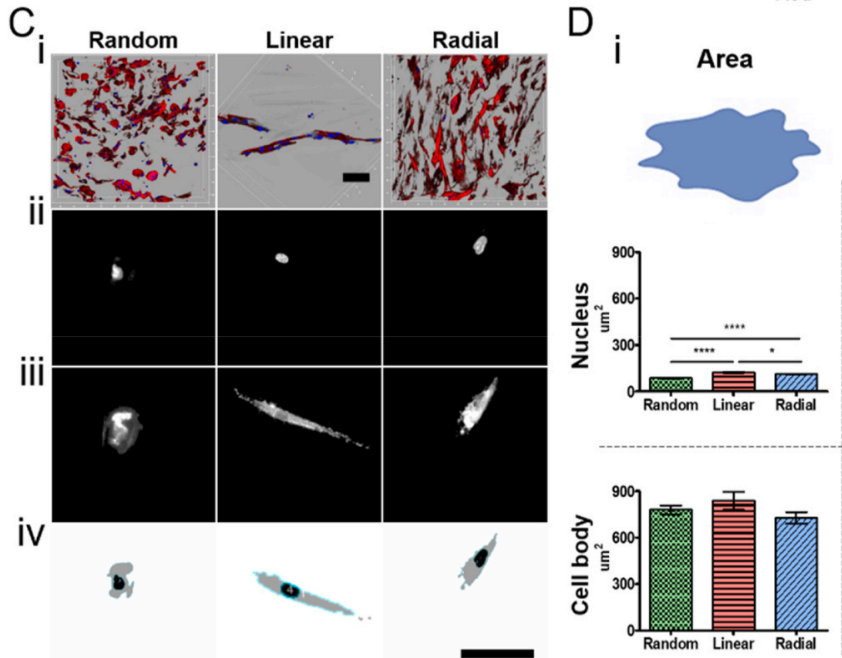

ii

Major axis

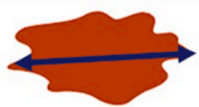

iii

Circularity

iV Aspect ratio
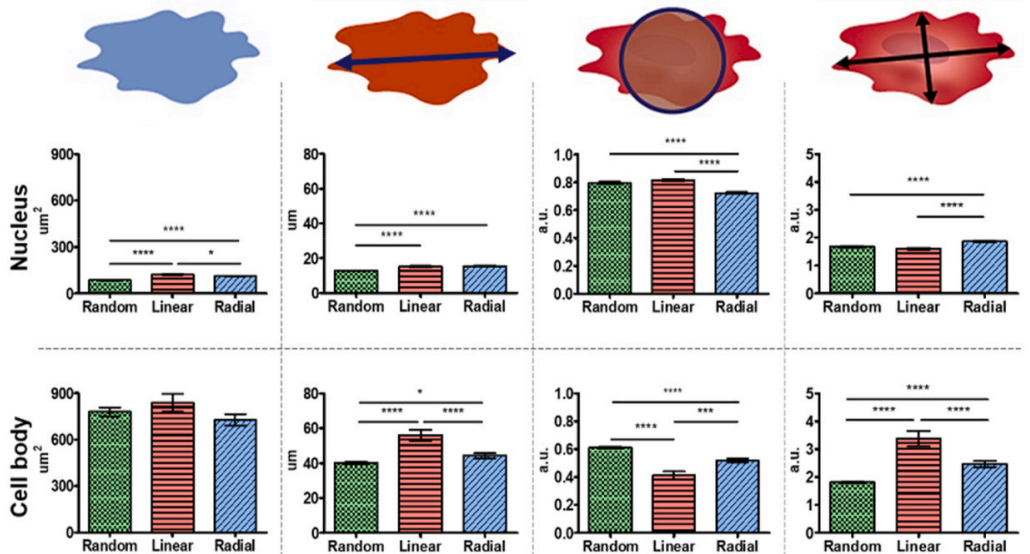

Fig. 5. Cell and nucleus orientation and shape dependence on matrix random, linear, and radial 3D orientation. (A) Macroscopic images of the cultured constructs were obtained and represented. (B) Human ASCs were cultured into the (i) Random, (ii) Linear, and (iii) Radial scaffolds architectures and imaged. ASCs (red) were stained using CellTrackerTM CM-DiI Dye and DNA (blue) immunostained with DAPI for nuclei observation. Cell orientation was assessed with FIJI 'Directionality' tool and plotted $(\mathrm{N}=5$, scale bar $=100 \mu \mathrm{m})$. (C i) Cell distribution and shape were 3D reconstructed with confocal microscopy. Isolated (C ii) nuclei and (C iii) cell body were identified (C iv) for morphological characterization using FIJI 'Analyze Particles' tool (scale bar $=100 \mu \mathrm{m}$ ). (D) Nucleus and cell body morphological characterization was represented with (D i) area, (D ii) major axis, (D iii) circularity, and (D iv) aspect ratio. Data are shown as Mean \pm SEM. Statistical significance is represented as ${ }^{*} \mathrm{p}<0.05,{ }^{* * *} \mathrm{p}<0.0002,{ }^{* * * *} \mathrm{p}<0.0001$. (For interpretation of the references to colour in this figure legend, the reader is referred to the Web version of this article.) 
orientation was predominantly stained with red and pink colors, while radial orientations was demonstrated with blue or green ones, according to the colour wheel on the right side of Fig. 4.

To assess the pore geometry, cross-sections along the Z-axis were performed at three different heights in the different formulations (Fig. 4C), showing the control over directionality obtained when applying the developed ice-templating strategy in cryogels. Importantly, the authors observed a better experimental outcome/control when fabricating linear-radial and linear-radial-linear architecture than the radial-linear-radial one.

\subsection{Cell and nucleus orientation and shape in response to matrix architecture}

To understand how the fabricated architectures would influence human cell behavior, human adipose-derived stem cells (hASCs) from Hoffa's body were cultured into the random, linear, and radial porous arrangements. Macroscopic images of the cultured constructs were obtained and represented in Fig. 5A. Cell morphology and arrangement at the microscale were assessed, showing different shapes depending on the scaffolds architecture (Fig. 5B-i, ii, and iii, respectively). Fig. 5B also demonstrated that cell orientation was directed by 3D porosity. Cell proliferation in the different architectures was represented in figure S4, showing higher cell proliferation in the condition of radial architecture. This result was also experimentally observed since the conditions of radial and random architectures demonstrated larger medium and cell retention during seeding than the linear one. Additionally, cell nuclei and cytoskeleton morphologies were assessed (Fig. 5C), demonstrating that nucleus $\left(124 \mu \mathrm{m}^{2}\right)$ and cytoskeleton $\left(837 \mu \mathrm{m}^{2}\right)$ areas were slightly increased in linear architecture (Fig. 5D-i), also reflected in the respective major axis (nucleus: $16 \mu \mathrm{m}$ in linear porosity, $18 \mu \mathrm{m}$ in radial porosity; cytoskeleton: $56 \mu \mathrm{m}$ in linear porosity), as represented in Fig. 5D-ii. Regarding shape descriptors, nuclei were more elongated in the radial than other evaluated architectures. Cell bodies were more circular in random and elongated in linear architectures, as demonstrated by circularity (Fig. 5D-iii) and aspect ratio assessments (Fig. 5D-iv).

\subsection{Hepatic biomarkers expression from cell co-cultures in random, linear, and radial $3 D$ architectures}

As a final goal, hASCs and human umbilical vein endothelial cells (hUVECs) were co-cultured under hepatogenic conditions into the different porous architectures. The influence of porous architectures in cell orientation was also shown in co-cultures, as represented in Fig. 6A-i. Cell orientation was also quantified (Fig. 6A-ii), showing the distribution frequency of cellular angle in the three porous arrangements. hASCs co-cultured with hUVECs in the random scaffolds showed no peak of orientation, meaning an isotropic cellular orientation, while the linear and radial scaffolds directed cells to organize anisotropically, as demonstrated by the peak at $\approx 0^{\circ}$ and $\approx 60^{\circ}$, respectively.

Furthermore, the endothelial segments formed by hUVECs were assessed, as represented in Fig. 6A-iii. The formation of segments, meshes, and branches was plotted, showing the formed networks in random, linear, and radial architectures.

To study the correlation between cellular arrangement and phenotypic variations hepatic biomarkers were assessed [3]. Specifically, cytochrome P450 (CYP450) activity (health biomarker [33,34]), osteopontin (OPN, cirrhosis biomarker [35]), and endothelial network, which is affected by liver health and fibrotic conditions [36] were assessed (Fig. 6B-i). The area occupied by cells at day 21 was also assessed in the three different architectures, showing significantly increased confluency in random and radial scaffolds $(\mathrm{p}<0.05$; Fig. 6B-ii). The endothelial network was analyzed through the quantification of master segments, meshes, and branches (Fig. 6B-iii). While master segments of endothelial cells were predominantly present in the linear organization, meshes and branches were significantly increased in the radial architecture $(\mathrm{p}<0.005)$.

OPN was immunostained in the co-cultured cells per conditions (Fig. 6B-iv), and its presence was assessed in the different architectures. Although a slight increase was observed in the linear condition, no significant differences were noted (Fig. 6B-v). The hepatic CYP450 indicator showed a significantly increased activity of the cytochrome by cells cultured in radial compared to the linear $(\mathrm{p}<0.001)$ and random (p $<0.005$ ) arrangements (Fig. 6B-vi).

Importantly, the authors consider that an accurate analysis of the biological results observed in Fig. 6 indicates that the different architectures induce varied cellular confluence and organization, which seem to influence CYP450 activity (normalized data), rather than a direct correlation between the macro-architecture and the presence or activity of these biomarkers (osteopontin expression and CYP450 activity).

\section{Discussion}

In the present work, the main aim was to develop and study a new system and methodology to enable the coding of multidirectional pore orientation into cryogels. Beyond the potential for broader applications, the disclosed results reveal the fabricated multidirectional structures composed of architectures with directed linear and radial pore orientations and combinations thereof. The effect of the 3D porous architectures on cell morphology, hepatic biomarkers, and the endothelial network is herein demonstrated.

First, the 3DICE, an ice-templating system, was developed. Icetemplating has been reported in the state-of-the-art as a top-down fabrication method. So far, diverse ice-templating strategies and devices were reported for varied applications, which are limited in controlling multidirectional pore orientation and 3D-coded porosity. While Pawelec et al. (2015) and Moreira et al. [37] developed devices to control pore size and geometry by applying ranges of freezing temperatures [26], Stolze et al. (2016) produced porous electrodes for organic batteries by an ice-templating approach, revealing that the pores were well structured and aligned in parallel [38]. Following the same rationale, Naviroj et al. (2017) recently disclosed a metal mold to produce scaffolds composed of long linear pores [24], which slightly revealed the potential use of ice-templating to organize porosity in more than one axis. In this trend, Tang et al. (2016) demonstrated that multiple pore orientation could be achieved in the horizontal plane of the cryogel [39], and Zhang et al. (2020) showed that wettability can be used to better control aligned and circular lamellar plate growth [40]. However, there are no reports of directional coding of pores in multiple orientation at specific regions of a single structure. So, the urge for multidirectional living tissue engineering led to the development of multiple alternative strategies to control hydrogels 3D arrangement, such as 3D printing [41] or bioprinting [42,43], and magnetic assembling [44] as the most promising fabrication mechanisms. Although the control over 3D hydrogel arrangement is the main advantage of these techniques over the state-of-the-art of ice-templating, fiber fusion, poor control at the nano- and micro-scale, as well as structure collapsing are the main drawbacks still to be solved. In the present work, the controlled formation of specific architectures was demonstrated with two and three pore geometry/orientations towards multiple axis. This way, a multidirectional control over porosity architecture was obtained using 3DICE to improve ice-templating, fabricating porosity with controlled orientation into horizontal and vertical axis at the sub-millimeter scale for the first time, with pores ranging from $64 \mathrm{~nm}$ to $454 \mathrm{~nm}$.

Strikingly, storage modulus and $\tan \delta$ were proven to directly depend on 3D pore architecture, while keeping the same polymeric content. More specifically, the storage modulus increases from random and radial pore orientation to the vertical one, meaning that when the applied force vector has the same orientation of the porosity, the required energy to destroy the structure is the highest. On the other hand, the $\tan \delta$ decreases inversely proportional to the storage modulus, demonstrating 
A

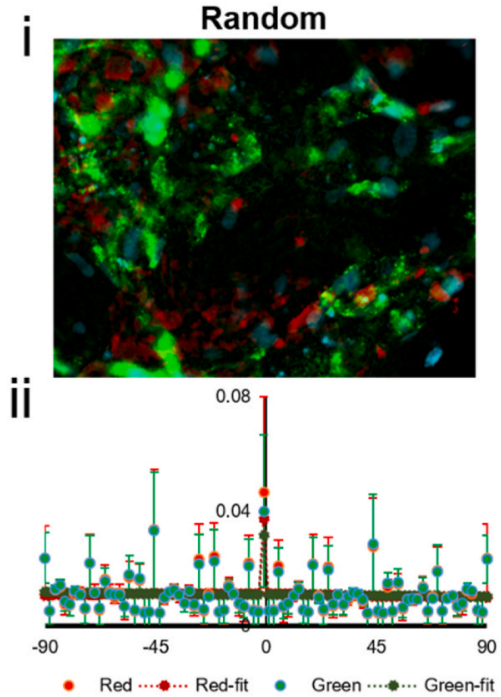

iii

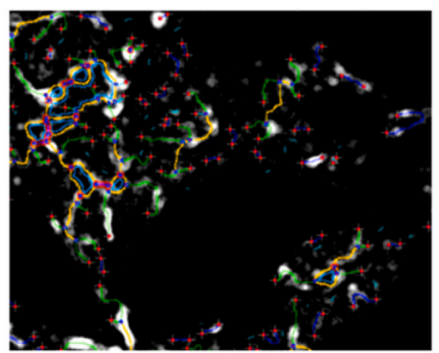

$\mathrm{B}_{\mathrm{i}}$

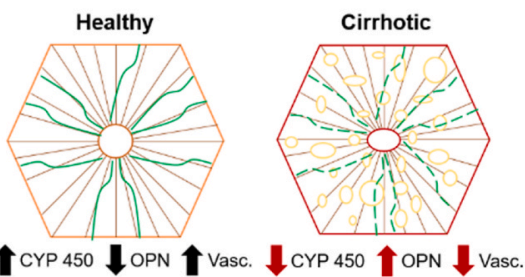

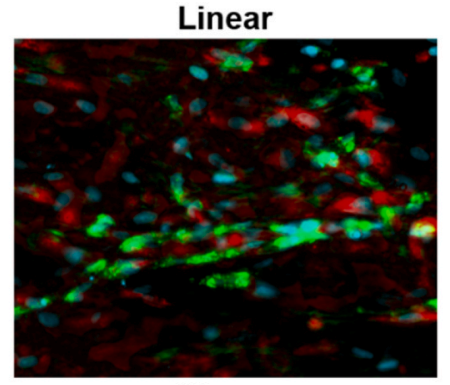

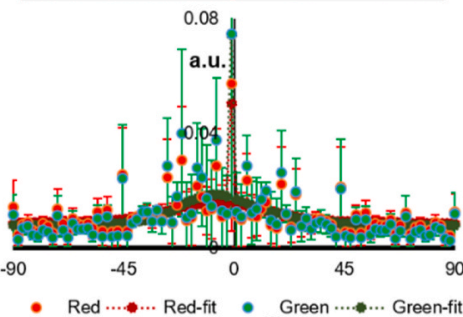

Degree $\left({ }^{\circ}\right)$

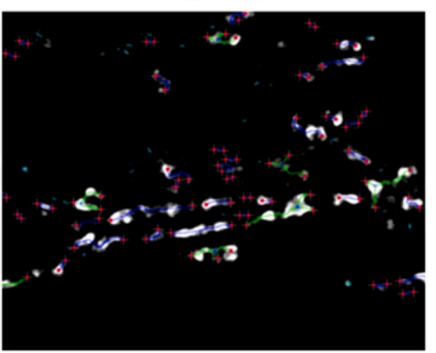

ii

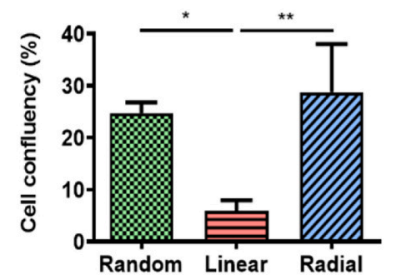

Radial
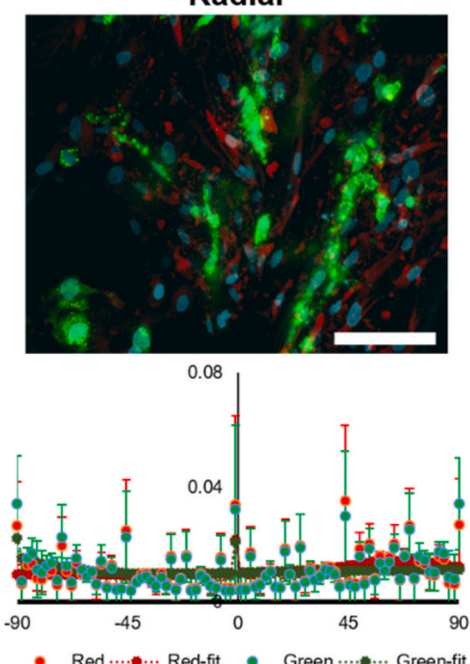

- Red ....... Red-fit • Green ....... Green-fit

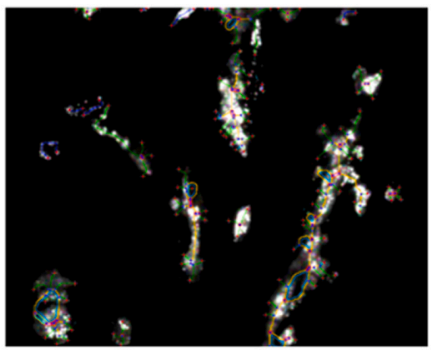

iii

\section{Endothelial Network}

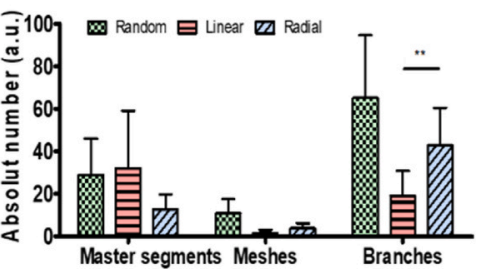

vi

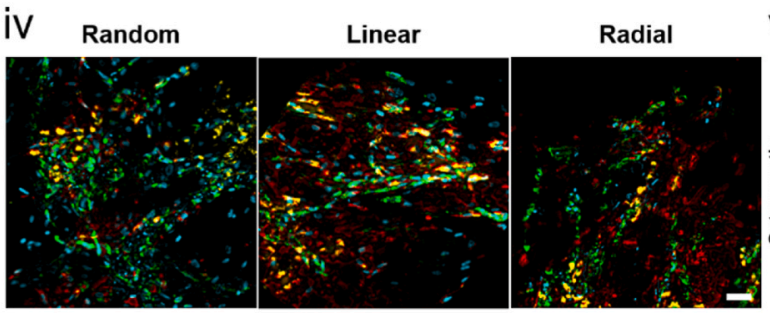

$\mathrm{V}$

Osteopontin
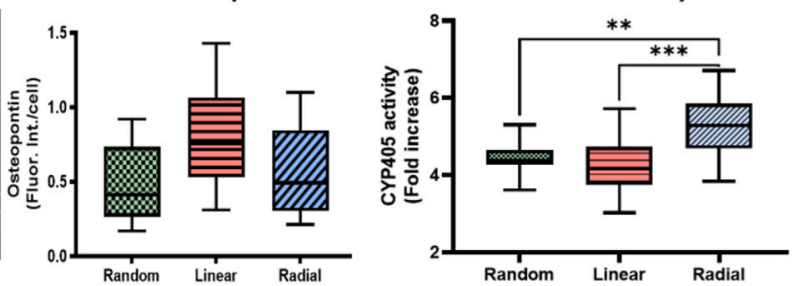

Fig. 6. Co-cultured cell orientation, endothelial networks formation, and hepatic biomarkers expression in random, linear, and radial 3D architectures. (A i) Co-cultures of hASCs and hUVECs were performed and the cellular orientation of stem (red) and endothelial (green) cells were plotted. ASCs (red) were stained using CellTrackerTM CM-DiI Dye, while hUVECs (green) were positively expressing Green Fluorescent Protein (GFP). (A ii) hASCs and hUVECs orientations in the three different architectures were plotted (Data are shown as Mean $\pm \mathrm{SD} ; \mathrm{N}=6$; scale bar $=50 \mu \mathrm{m}$ ). (A iii) The formed endothelial network in the different architectures was analyzed per condition. The tubular segments were identified in the microscopic images using the Angiogenesis Analyzer tool [32] for FIJI [31]. Yellow lines represent the master segments, tubes that connect together to different junctions; green lines represent branches; light blue surrounding the master segment is the mesh; dark blue represents isolated tubes; red circles represent the master junction points. (B i) Hepatic biomarkers were assessed, namely the CYP450 (health biomarker) and osteopontin (OPN, cirrhosis biomarker), as well as the quantification of the endothelial network. (B ii) The area occupied by the population of cells was also quantified, represented as cell confluence per condition. (B iii) The endothelial major segments, meshes, and branches were quantified per architecture and plotted. (B iv) OPN protein (hASCs are red, hUVECs green, OPN yellow) was assessed in the co-cultures applying anti-OPN immunostaining (scale bar $=50$ ) and (B v) quantified with FIJI $(\mathrm{N}=6$ ). (B vi) The CYP450 activity was quantified using the Cytochrome P450 Reductase Activity Assay Kit on day 21 ( $\mathrm{N}=7$ ). Data shown as Mean \pm SEM $\left(* \mathrm{p}<0.05,{ }^{* *} \mathrm{p}<0.005,{ }^{* * *} \mathrm{p}<0.001\right)$. (For interpretation of the references to colour in this figure legend, the reader is referred to the Web version of this article.) 
that the recovery of the shape is enhanced by a $90^{\circ}$ pore orientation relative to the applied force vector. This observation was previously described in the literature. Torres-Sanchez et al. (2018) reported that high aspect ratio pores loaded perpendicularly to the alignment direction, weakened the structure when compared to spheroidal voids for equivalent volumetric porosities [45]. Additionally, Hyun et al. (2004) compared the parallel and vertical tensile stress of linear porosity, concluding that both yield and ultimate tensile strength of specimens with cylindrical pore orientation parallel to the tensile direction are much higher than that perpendicular one [46], while Seuba et al. (2016) and Canadas et al. (2018) showed that isotropic structures have lower strength than the anisotropic ones $[6,47]$. The parallel alignment of the walls with the load allowed an optimal distribution of the stresses and prevented the bending of the struts at lower loads. Here, we confirmed these observations using a structure produced in the 3DICE from a cryogel crosslinked using visible light as the trigger. Beyond the reported results, we additionally correlated the radial pore orientation with the mechanical response to compressive stress (low storage modulus, high tan $\delta$ ).

Then, the ability to code pore orientation within multidirectional 3D architectures was tested by intercalating thermally conductive and insulating plates around the hydrogel mold in the 3DICE. Interestingly, we found that double- and triple-multidirectional pore orientations were obtained with the following configurations: Linear-Radial, LinearRadial-Linear, and Radial-Linear-Radial pore orientation along with the structure Z-axis. In previous studies [15], the conductivity of the surrounding material was tested to obtain specific pore orientation and shape. However, the fabrication of multidirectional structures composed of specific pore orientations along the Z-axis was described for the first time in the present study, using the 3DICE to obtain a continuous structure composed of pores coded into multiple orientations, and unlocking further potential for the ice-templating top-down fabrication methodology. This way, not only specific porous architectures are possible to obtain in 3D cryogels, but also specific mechanical properties are tuned along the Z-axis by correlation with the results observed using the dynamic mechanical analysis described above.

Finally, the produced structures were tested with different cell types in single- and co-cultures. hASCs single cultures were used to study cell distribution and morphology, while the co-culture of hASCs and hUVECs was assessed under hepatic differentiation process. The influence of random, linear, and radial 3D architectures was demonstrated, having a direct effect on cellular distribution and shape, guided by the different artificial ECM 3D arrangements, as previously observed in 2D [48]. Importantly, previous works reported that macro-architectural cues impact tissue infiltration, but also liver lobules enlargement during ontogeny [10], SOX-9 presence in osteo-chondrogenesis [6], PSD-95 post-synaptic biomarker in cortex neural models [15], and lobular adipose tissue influences progenitor cells fate [49]. Bone marrow mesenchymal stem cell surface marker CD105 expression was also shown transient depending on the matrix architecture [50]. Still, there is a wide gap about the influence of macro-architecture over biological function. Strikingly, the hepatic lobules, focused in the present work as a proof-of-concept, present a specific ECM architecture, as a radially organized tissue $[8,10]$, and the cell shape and size is controlled by pore orientation, as herein demonstrated. Moreover, while endothelial segments were in larger number in the linear architecture, branches and meshes were more present in the random and radial conditions compared to linear porosity.

Additionally, CYP450 activity was significantly increased in the hepatic differentiating hASCs when cultured in the radial pore architecture, compared to the linear one, which activity is an indicator of liverspecific function to assess the utility for drug metabolism, as previously observed in 2D [8]. Interestingly, in a previous study, the liver function was demonstrated to be more dependent on lobule architecture than number [10]. However, in the present study the authors consider that the macro-architecture is not the only factor directly inducing different
CYP450 activity, but also the cell confluence and organization are impacted by these architectures, influencing the cytochrome activity. Finally, human OPN, a cirrhotic biomarker [35] and important hypertrophic indicator, was more expressed by cells cultured into the linear pore orientation compared to the radial and random ones, which can be indirectly correlated with the observed cellular macro-organization of the natural tissue [8], as previously referred. Overall, a very different biologic performance, namely cell adhesion, morphology, and co-culture cellular distribution, was observed in the different macro-architectures. For instance, higher cell confluence per surface area was observed in the random and radial porous structures than in the linear ones, which is of high importance for in vitro and in vivo scaffold performance. Similarly, a higher number of endothelial meshes and branches were present in these same conditions, revealing a relevant indication for future vascularization studies. Although these results are not related to specific biomarkers of the hepatic model, they seriously impact engineered tissues, in general. Moreover, the hepatic lobule functionality is significantly dependent on its vascularization, as demonstrated by Debbaut et al. [51]. These lobuli vary in diameter from 1 to $2.5 \mathrm{~mm}$, and the vascular septa and the anisotropic permeability of the tissue are determinant factors to mimic unphysiological and pathological conditions. Thus, the future development of liver disease in vitro models is supported by the outcomes of the present study, which demonstrates the impact of 3D architectures in the hepatic biomarker, but triggers the attention for further studies on tissue macro-architecture and biomarker correlation.

As the main limitations, the obtained porosity of the fabricated structures is randomly formed at the nanoscale level, requiring future studies to improve the control over pore interconnectivity. Moreover, the obtained layers of coded pore orientation are limited in thickness, reaching a minimum of around $1.5-2 \mathrm{~mm}$ (still, in the range of the native hepatic lobuli). In the future, solutions to overcome the described limitations will be tested, namely by adapting the thermal reactivity/conductivity of the gel.

\section{Conclusions}

The main aim of the present work was to develop a simple, costefficient device to control 3D ice-templating in cryogels. More specifically, the long-range control over pore multidirectional orientation in water-based systems. In summary, the fabrication of continuous multidirectional porosity sequentially coded by the 3DICE through 3D cryogels was demonstrated. Beyond the simplicity of the device and fabrication strategy, the disclosed method enables the control over pore size, shape, and formation of multiple 3D porous architectures into three main orientations, random/isotropic, linear, and radial, adaptable to large-scale applications. Importantly, the method has broad utility in diverse fields of the materials engineering area and unlocks the potential of ice-templating to a different level than the technique's current stateof-the-art. In the present study, we focused on the biological use for tissue engineering and in vitro hepatic models, demonstrating that matrix macro-architecture controls cell distribution and morphology, which impacts its phenotype by direct correlation of cell and nuclei shape, hASCs orientation, and endothelial network, as well as CYP activity and osteopontin presence. Overall, linear orientation was less efficient to reproduce a biomimetic hepatic tissue in vitro, while radial cellular organization not only improved endothelization branches, but also the expression of CYP450, in co-cultures. Overall, from matrix shape to cellular phenotype, the present work reports a step forward in the control of ice-templating, at the millimeter to centimeter range, to fabricate scaffolds for tissue engineering, raising the methodology to a sophisticated level of multiple possibilities, increasing the potential of the technique, and proving its importance to engineer hierarchical tissues. 


\section{Credit author statement}

Raphaël F. Canadas: Conceptualization, Methodology, Data curation, Investigation; Writing. João B. Costa: Investigation (mechanical analysis), Data curation, Writing; Zhengwei Mao: Funding acquisition, Project administration; Changyou Gao: Funding acquisition, Project administration; Utkan Demirci: Conceptualization; Rui L. Reis: Funding acquisition, Resources; Alexandra P. Marques: Supervision, Writing; Joaquim M. Oliveira: Funding acquisition, Supervision, Writing, Project administration.

\section{Declaration of competing interest}

Dr. Raphaël F. Canadas (RC) is a founder of and has an equity interest in: (i) Tech4MED ${ }^{\mathrm{TM}}$, a company that is developing therapeutic and diagnostic tools based on biomolecules and cells, (ii) LANDRATECH, Lda., a company exploring forest-derived biomaterials for diverse industrial applications. Prof. Utkan Demirci (UD) is a founder of and has an equity interest in: (i) DxNow Inc., a company that is developing microfluidic IVF tools and imaging technologies, (ii) Koek Biotech, a company that is developing microfluidic technologies for clinical solutions, (iii) Levitas Inc., a company focusing on developing microfluidic sorters using magnetic levitation, (iv) Hillel Inc., a company bringing microfluidic cell phone tools to home settings, and (v) Mercury Biosciences, a company focused on extracellular vesicles isolation tools. RC's and UD's interests were viewed and managed in accordance with the conflict of interest policies.

\section{Acknowledgments}

The authors are grateful for the Portuguese Foundation for Science and Technology (FCT) distinction attributed to R. F. Canadas (SFRH/ BD/92565/2013), and to J. M. Oliveira (IF/00423/2012, IF/01285/ 2015). R. F. Canadas is also thankful to FCT, Fundo Europeu de Desenvolvimento Regional (FEDER), and Programa Operacional Competitividade e Internacionalização (POCI) for funding the B-Liver Project (PTDC/EMD-EMD/29139/2017). The authors are also thankful to FCT for supporting the project Hierarchitech (M-ERA-NET/0001/2014) and for the funds provided under the 3 BioMeD project (JICAM/0001/2017). The authors acknowledge that this material and collaboration is based in part upon work supported by Luso-American Development Foundation (FLAD), 2016/CON15/CAN6). U. Demirci is also grateful for the Canary Center at Stanford for Cancer Early Detection Seed Award. The authors are also grateful for the support provided by Diana Bicho and Nicolas Cristini on scaffold characterization and cell culture, respectively.

\section{Appendix A. Supplementary data}

Supplementary data to this article can be found online at https://doi. org/10.1016/j.biomaterials.2021.121112.

\section{Data availability}

The raw/processed data required to reproduce these findings cannot be shared at this time as the data also forms part of an ongoing study.

\section{References}

[1] J. Barthes, H. Ozcelik, M. Hindie, A. Ndreu-Halili, A. Hasan, N.E. Vrana, Cell microenvironment engineering and monitoring for tissue engineering and regenerative medicine: the recent advances, BioMed Res. Int. 2014 (2014) 921905, https://doi.org/10.1155/2014/921905.

[2] W.-B. Tsai, Y.-C. Ting, J.-Y. Yang, J.-Y. Lai, H.-L. Liu, Fibronectin modulates the morphology of osteoblast-like cells (MG-63) on nano-grooved substrates, J. Mater. Sci. Mater. Med. 20 (2009) 1367-1378, https://doi.org/10.1007/s10856-0083687-8.
[3] W.-B. Tsai, J.-H. Lin, Modulation of morphology and functions of human hepatoblastoma cells by nano-grooved substrata, Acta Biomater. 5 (2009) 1442-1454, https://doi.org/10.1016/j.actbio.2009.01.002.

[4] U.A. Gurkan, R. El Assal, S.E. Yildiz, Y. Sung, A.J. Trachtenberg, W.P. Kuo, U. Demirci, Engineering anisotropic biomimetic fibrocartilage microenvironment by bioprinting mesenchymal stem cells in nanoliter gel droplets, Mol. Pharm. 11 (2014) 2151-2159, https://doi.org/10.1021/mp400573g.

[5] D.R. Pereira, R.F. Canadas, J. Silva-Correia, A. da Silva Morais, M.B. Oliveira, I. R. Dias, J.F. Mano, A.P. Marques, R.L. Reis, J.M. Oliveira, Injectable gellan-gum/ hydroxyapatite-based bilayered hydrogel composites for osteochondral tissue regeneration, Appl. Mater. Today. 12 (2018) 309-321, https://doi.org/10.1016/j. apmt.2018.06.005.

[6] R.F. Canadas, T. Ren, A.P. Marques, J.M. Oliveira, R.L. Reis, U. Demirci, Biochemical gradients to generate 3D heterotypic-like tissues with isotropic and anisotropic architectures, Adv. Funct. Mater. (2018), 1804148, https://doi.org/ 10.1002/adfm.201804148, 0 .

[7] A.J. Sophia Fox, A. Bedi, S.A. Rodeo, The basic science of articular cartilage: structure, composition, and function, Sport Health 1 (2009) 461-468, https://doi. org $/ 10.1177 / 1941738109350438$.

[8] C.-T. Ho, R.-Z. Lin, R.-J. Chen, C.-K. Chin, S.-E. Gong, H.-Y. Chang, H.-L. Peng, L. Hsu, T.-R. Yew, S.-F. Chang, C.-H. Liu, Liver-cell patterning Lab Chip: mimicking the morphology of liver lobule tissue, Lab Chip 13 (2013), https://doi.org/ 10.1039/c3lc50402f.

[9] D.M. Hoganson, H.I. Pryor 2nd, I.D. Spool, O.H. Burns, J.R. Gilmore, J.P. Vacanti, Principles of biomimetic vascular network design applied to a tissue-engineered liver scaffold, Tissue Eng. 16 (2010) 1469-1477, https://doi.org/10.1089/ten. TEA.2009.0118.

[10] V. Papp, K. Dezsö, V. László, P. Nagy, S. Paku, Architectural changes during regenerative and ontogenic liver growth in the rat, Liver Transplant. 15 (2009) 177-183, https://doi.org/10.1002/1t.21665.

[11] K.M. Yamada, E. Cukierman, Modeling tissue morphogenesis and cancer in 3D, Cell 130 (2007) 601-610, https://doi.org/10.1016/j.cell.2007.08.006.

[12] G. Rijal, W. Li, Native-mimicking in vitro microenvironment: an elusive and seductive future for tumor modeling and tissue engineering, J. Biol. Eng. 12 (2018) 20, https://doi.org/10.1186/s13036-018-0114-7.

[13] A.I. Teixeira, G.A. Abrams, P.J. Bertics, C.J. Murphy, P.F. Nealey, Epithelial contact guidance on well-defined micro- and nanostructured substrates, J. Cell Sci. 116 (2003), https://doi.org/10.1242/jcs.00383, 1881 LP - 1892.

[14] A. Tsuruma, M. Tanaka, S. Yamamoto, N. Fukushima, H. Yabu, M. Shimomura, Topographical control of neurite extension on stripe-patterned polymer films, Colloids Surfaces A Physicochem. Eng. Asp. 284-285 (2006) 470-474, https://doi. org/10.1016/j.colsurfa.2005.11.100.

[15] R.F. Canadas, T. Ren, A. Tocchio, A.P. Marques, J.M. Oliveira, R.L. Reis, U. Demirci, Tunable anisotropic networks for 3-D oriented neural tissue models, Biomaterials 181 (2018) 402-414, https://doi.org/10.1016/j. biomaterials.2018.07.055.

[16] P.-Y. Wang, J. Yu, J.-H. Lin, W.-B. Tsai, Modulation of alignment, elongation and contraction of cardiomyocytes through a combination of nanotopography and rigidity of substrates, Acta Biomater. 7 (2011) 3285-3293, https://doi.org/ 10.1016/j.actbio.2011.05.021.

[17] J.B. Recknor, J.C. Recknor, D.S. Sakaguchi, S.K. Mallapragada, Oriented astroglial cell growth on micropatterned polystyrene substrates, Biomaterials 25 (2004) 2753-2767, https://doi.org/10.1016/j.biomaterials.2003.11.045.

[18] A.S.G. Curtis, N. Gadegaard, M.J. Dalby, M.O. Riehle, C.D.W. Wilkinson, G. Aitchison, Cells react to nanoscale order and symmetry in their surroundings, IEEE Trans. NanoBioscience 3 (2004) 61-65, https://doi.org/10.1109/ TNB.2004.824276.

[19] A. Sørensen, T. Alekseeva, K. Katechia, M. Robertson, M.O. Riehle, S.C. Barnett, Long-term neurite orientation on astrocyte monolayers aligned by microtopography, Biomaterials 28 (2007) 5498-5508, https://doi.org/10.1016/j. biomaterials.2007.08.034.

[20] A. Béduer, C. Vieu, F. Arnauduc, J.-C. Sol, I. Loubinoux, L. Vaysse, Engineering of adult human neural stem cells differentiation through surface micropatterning, Biomaterials 33 (2012) 504-514, https://doi.org/10.1016/j. biomaterials.2011.09.073.

[21] T. Ren, B. Grosshäuser, K. Sridhar, T.J.F. Nieland, A. Tocchio, U. Schepers, U. Demirci, 3-D geometry and irregular connectivity dictate neuronal firing in frequency domain and synchronization, Biomaterials 197 (2019) 171-181, https:// doi.org/10.1016/j.biomaterials.2019.01.017.

[22] P. Zhuang, A.X. Sun, J. An, C.K. Chua, S.Y. Chew, 3D neural tissue models: from spheroids to bioprinting, Biomaterials 154 (2018) 113-133, https://doi.org/ 10.1016/j.biomaterials.2017.10.002

[23] A. Arslan-Yildiz, R. El Assal, P. Chen, S. Guven, F. Inci, U. Demirci, Towards artificial tissue models: past, present, and future of 3D bioprinting, Biofabrication 8 (2016) 14103, https://doi.org/10.1088/1758-5090/8/1/014103.

[24] M. Naviroj, M.M. Wang, M.T. Johnson, K.T. Faber, Nucleation-controlled freeze casting of preceramic polymers for uniaxial pores in Si-based ceramics, Scripta Mater. 130 (2017) 32-36, https://doi.org/10.1016/j.scriptamat.2016.10.038.

[25] Y. Tang, Q. Miao, S. Qiu, K. Zhao, L. Hu, Novel freeze-casting fabrication of aligned lamellar porous alumina with a centrosymmetric structure, J. Eur. Ceram. Soc. 34 (2014) 4077-4082, https://doi.org/10.1016/j.jeurceramsoc.2014.05.040.

[26] K.M. Pawelec, A. Husmann, S.M. Best, R.E. Cameron, Altering crystal growth and annealing in ice-templated scaffolds, J. Mater. Sci. 50 (2015) 7537-7543, https:// doi.org/10.1007/s10853-015-9343-z.

[27] D. Bicho, R.F. Canadas, C. Gonçalves, S. Pina, R.L. Reis, J.M. Oliveira, Porous aligned $\mathrm{ZnSr}$-doped $\beta$-TCP/silk fibroin scaffolds using ice-templating method for 
bone tissue engineering applications, J. Biomater. Sci. Polym. Ed. (2021) 1-17, https://doi.org/10.1080/09205063.2021.1952382.

[28] H. Bai, Y. Chen, B. Delattre, A.P. Tomsia, R.O. Ritchie, Bioinspired large-scale aligned porous materials assembled with dual temperature gradients, Sci. Adv. 1 (2015), e1500849, https://doi.org/10.1126/sciadv.1500849.

[29] R.F. Canadas, P. Patrício, V. Brancato, L. Gasperini, D. Caballero, R.A. Pires, J. B. Costa, H. Pereira, P. Yong, L.P. da Silva, J. Chen, S.C. Kundu, N.A.M. Araújo, R. L. Reis, A.P. Marques, J.M. Oliveira, Convection patterns gradients of non-living and living micro-entities in hydrogels, Appl. Mater. Today. 21 (2020), https://doi. org/10.1016/j.apmt.2020.100859.

[30] C.S. Bahney, T.J. Lujan, C.W. Hsu, M. Bottlang, J.L. West, B. Johnstone, Visible light photoinitiation of mesenchymal stem cell-laden bioresponsive hydrogels, Eur Cell. Mater. 22 (2011) 43-55, https://doi.org/10.22203/ecm.v022a04, discussion 55.

[31] J. Schindelin, I. Arganda-Carreras, E. Frise, V. Kaynig, M. Longair, T. Pietzsch, S. Preibisch, C. Rueden, S. Saalfeld, B. Schmid, J.-Y. Tinevez, D.J. White, V. Hartenstein, K. Eliceiri, P. Tomancak, A. Cardona, Fiji: an open-source platform for biological-image analysis, Nat. Methods 9 (2012) 676, https://doi.org/ 10.1038/nmeth.2019.

[32] G. Carpentier, Contribution: Angiogenesis Analyzer, ImageJ News, 2012. https://i magej.nih.gov/ij/notes.html. (Accessed 9 September 2018).

[33] C.D. Fisher, A.J. Lickteig, L.M. Augustine, J. Ranger-Moore, J.P. Jackson, S. S. Ferguson, N.J. Cherrington, Hepatic cytochrome P450 enzyme alterations in humans with progressive stages of nonalcoholic fatty liver disease, Drug Metab. Dispos. 37 (2009) 2087-2094, https://doi.org/10.1124/dmd.109.027466.

[34] R.F. Frye, N.K. Zgheib, G.R. Matzke, D. Chaves-Gnecco, M. Rabinovitz, O.S. Shaikh, R.A. Branch, Liver disease selectively modulates cytochrome P450-mediated metabolism, Clin. Pharmacol. Ther. 80 (2006) 235-245, https://doi.org/10.1016/ j.clpt.2006.05.006.

[35] Z. Khajehahmadi, S. Mohagheghi, S. Nikeghbalian, B. Geramizadeh, I. Khodadadi, J. Karimi, H. Tavilani, Liver stiffness correlates with serum osteopontin and TAZ expression in human liver cirrhosis, Ann. N. Y. Acad. Sci. 1465 (2020) 117-131, https://doi.org/10.1111/nyas.14259.

[36] I. Amat-Roldan, A. Berzigotti, R. Gilabert, J. Bosch, Assessment of hepatic vascular network connectivity with automated graph analysis of dynamic contrastenhanced US to evaluate portal hypertension in patients with cirrhosis: a pilot study, Radiology 277 (2015) 268-276, https://doi.org/10.1148/ radiol.2015141941.

[37] H.R. Moreira, L.P. da Silva, R.L. Reis, A.P. Marques, Tailoring gellan gum spongylike hydrogels' microstructure by controlling freezing parameters, Polymers 12 (2020) 329, https://doi.org/10.3390/polym12020329.

[38] C. Stolze, T. Janoschka, S. Flauder, F.A. Müller, M.D. Hager, U.S. Schubert, Investigation of ice-templated porous electrodes for application in organic batteries, ACS Appl. Mater. Interfaces 8 (2016) 23614-23623, https://doi.org/ 10.1021/acsami.6b05018.

[39] Y. Tang, S. Qiu, Q. Miao, C. Wu, Fabrication of lamellar porous alumina with axisymmetric structure by directional solidification with applied electric and magnetic fields, J. Eur. Ceram. Soc. 36 (2016) 1233-1240, https://doi.org/ 10.1016/j.jeurceramsoc.2015.12.012.

[40] N. Zhao, M. Li, H. Gong, H. Bai, Controlling ice formation on gradient wettability surface for high-performance bioinspired materials, Sci. Adv. 6 (2020), https://doi. org/10.1126/sciadv.abb4712 eabb4712.

[41] B. Derby, Printing and prototyping of tissues and scaffolds, Science (80) (2012) 338, https://doi.org/10.1126/science.1226340, 921 LP - 926.

[42] K. Jakab, A. Neagu, V. Mironov, R.R. Markwald, G. Forgacs, Engineering biological structures of prescribed shape using self-assembling multicellular systems, Proc. Natl. Acad. Sci. U. S. A 101 (2004) 2864-2869, https://doi.org/10.1073/ pnas.0400164101.

[43] H.-W. Kang, S.J. Lee, I.K. Ko, C. Kengla, J.J. Yoo, A. Atala, A 3D bioprinting system to produce human-scale tissue constructs with structural integrity, Nat. Biotechnol. 34 (2016) 312, https://doi.org/10.1038/nbt.3413.

[44] X. Feng, W.C. Max, R. Venkatakrishnan, F.T. Dylan, K.H. Onur, S. Yuree, L. Baoqiang, G.U. Atakan, U. Demirci, F. Xu, C.-A.M. Wu, V. Rengarajan, T. D. Finley, H.O. Keles, Y. Sung, B. Li, U.A. Gurkan, U. Demirci, Three-dimensional magnetic assembly of microscale hydrogels, Adv. Mater. 23 (2011) 4254-4260, https://doi.org/10.1002/adma.201101962.

[45] C. Torres-Sanchez, J. McLaughlin, R. Bonallo, Effect of pore size, morphology and orientation on the bulk stiffness of a porous Ti35Nb4Sn alloy, J. Mater. Eng. Perform. 27 (2018) 2899-2909, https://doi.org/10.1007/s11665-018-3380-0.

[46] S.-K. Hyun, T. Ikeda, H. Nakajima, Fabrication of lotus-type porous iron and its mechanical properties, Sci. Technol. Adv. Mater. 5 (2004) 201-205, https://doi. org/10.1016/j.stam.2003.11.005.

[47] J. Seuba, S. Deville, C. Guizard, A.J. Stevenson, Mechanical properties and failure behavior of unidirectional porous ceramics, Sci. Rep. 6 (2016) 24326, https://doi. org/10.1038/srep24326.

[48] G. Maheshwari, G. Brown, D.A. Lauffenburger, A. Wells, L.G. Griffith, Cell adhesion and motility depend on nanoscale RGD clustering, J. Cell Sci. 113 (2000) 1677. LP - 1686, http://jcs.biologists.org/content/113/10/1677.abstract.

[49] D. Estève, N. Boulet, C. Belles, A. Zakaroff-Girard, P. Decaunes, A. Briot, Y. Veeranagouda, M. Didier, A. Remaury, J.C. Guillemot, S. Ledoux, C. Dani, A. Bouloumié, J. Galitzky, Lobular architecture of human adipose tissue defines the niche and fate of progenitor cells, Nat. Commun. 10 (2019) 2549, https://doi.org/ 10.1038/s41467-019-09992-3.

[50] R.F. Canadas, J.M.B.T. Cavalheiro, J.D.T. Guerreiro, M.C.M.D. de Almeida, E. Pollet, C.L. da Silva, M.M.R. da Fonseca, F.C. Ferreira, Polyhydroxyalkanoates: waste glycerol upgrade into electrospun fibrous scaffolds for stem cells culture, Int. J. Biol. Macromol. 71 (2014) 131-140, https://doi.org/10.1016/j. ijbiomac. 2014.05.008.

[51] C. Debbaut, J. Vierendeels, J.H. Siggers, R. Repetto, D. Monbaliu, P. Segers, A 3D porous media liver lobule model: the importance of vascular septa and anisotropic permeability for homogeneous perfusion, Comput. Methods Biomech. Biomed. Eng. 17 (2014) 1295-1310, https://doi.org/10.1080/10255842.2012.744399. 